\title{
At the roots of dictionary compression: String attractors
}

\author{
Kempa, Dominik; Prezza, Nicola
}

Published in:

Proceedings of the 50th Annual ACM SIGACT Symposium on Theory of Computing

Link to article, DOI:

$10.1145 / 3188745.3188814$

Publication date:

2018

Document Version

Publisher's PDF, also known as Version of record

Link back to DTU Orbit

Citation (APA):

Kempa, D., \& Prezza, N. (2018). At the roots of dictionary compression: String attractors. In Proceedings of the 50th Annual ACM SIGACT Symposium on Theory of Computing (pp. 827-40). Association for Computing Machinery. https://doi.org/10.1145/3188745.3188814

\section{General rights}

Copyright and moral rights for the publications made accessible in the public portal are retained by the authors and/or other copyright owners and it is a condition of accessing publications that users recognise and abide by the legal requirements associated with these rights.

- Users may download and print one copy of any publication from the public portal for the purpose of private study or research.

- You may not further distribute the material or use it for any profit-making activity or commercial gain

- You may freely distribute the URL identifying the publication in the public portal 


\section{At the Roots of Dictionary Compression: String Attractors}

\author{
Dominik Kempa \\ Department of Computer Science, University of Helsinki \\ Helsinki, Finland \\ dkempa@cs.helsinki.fi
}

\author{
Nicola Prezza \\ DTU Compute, Technical University of Denmark \\ Kongens Lyngby, Denmark \\ Department of Computer Science, University of Pisa \\ Pisa, Italy \\ nicola.prezza@di.unipi.it
}

\begin{abstract}
A well-known fact in the field of lossless text compression is that high-order entropy is a weak model when the input contains long repetitions. Motivated by this fact, decades of research have generated myriads of so-called dictionary compressors: algorithms able to reduce the text's size by exploiting its repetitiveness. Lempel-Ziv 77 is one of the most successful and well-known tools of this kind, followed by straight-line programs, run-length Burrows-Wheeler transform, macro schemes, collage systems, and the compact directed acyclic word graph. In this paper, we show that these techniques are different solutions to the same, elegant, combinatorial problem: to find a small set of positions capturing all distinct text's substrings. We call such a set a string attractor. We first show reductions between dictionary compressors and string attractors. This gives the approximation ratios of dictionary compressors with respect to the smallest string attractor and allows us to uncover new asymptotic relations between the output sizes of different dictionary compressors. We then show that the $k$-attractor problem deciding whether a text has a size- $t$ set of positions capturing all substrings of length at most $k-$ is NP-complete for $k \geq 3$. This, in particular, includes the full string attractor problem. We provide several approximation techniques for the smallest $k$-attractor, show that the problem is APX-complete for constant $k$, and give strong inapproximability results. To conclude, we provide matching lower and upper bounds for the random access problem on string attractors. The upper bound is proved by showing a data structure supporting queries in optimal time. Our data structure is universal: by our reductions to string attractors, it supports random access on any dictionary-compression scheme. In particular, it matches the lower bound also on LZ77, straight-line programs, collage systems, and macro schemes, and therefore essentially closes (at once) the random access problem for all these compressors.
\end{abstract}

\section{CCS CONCEPTS}

- Theory of computation $\rightarrow$ Data compression; Packing and covering problems; Problems, reductions and completeness; Cell probe models and lower bounds;

Permission to make digital or hard copies of all or part of this work for personal o classroom use is granted without fee provided that copies are not made or distributed for profit or commercial advantage and that copies bear this notice and the full citation on the first page. Copyrights for components of this work owned by others than ACM must be honored. Abstracting with credit is permitted. To copy otherwise, or republish to post on servers or to redistribute to lists, requires prior specific permission and/or a fee. Request permissions from permissions@acm.org.

STOC'18, fune 25-29, 2018, Los Angeles, CA, USA

(C) 2018 Association for Computing Machinery.

ACM ISBN 978-1-4503-5559-9/18/06 ..\$15.00

https://doi.org/10.1145/3188745.3188814
Dictionary compression, compressed data structures

\section{ACM Reference Format:}

Dominik Kempa and Nicola Prezza. 2018. At the Roots of Dictionary Compression: String Attractors. In Proceedings of 50th Annual ACM SIGACT Symposium on the Theory of Computing (STOC'18). ACM, New York, NY, USA, 14 pages. https://doi.org/10.1145/3188745.3188814

\section{INTRODUCTION}

The goal of lossless text compression is to reduce the size of a given string by exploiting irregularities such as skewed character distributions or substring repetitions. Unfortunately, the holy grail of compression - Kolmogorov complexity [28] - is non-computable: no Turing machine can decide, in a finite number of steps, whether a given string has a program generating it whose description is smaller than some fixed value $K$. This fact stands as the basis of all work underlying the field of data compression: since we cannot always achieve the best theoretical compression, we can at least try to approximate it. In order to achieve such a goal, we must first find a model that captures, to some good extent, the degree of regularity of the text. For example, in the case of the text generated by a Markovian process of order $k$, the $k$-th order entropy $H_{k}$ of the source represents a lower bound for our ability to compress its outputs. This concept can be extended to that of empirical entropy [13] when the underlying probabilities are unknown and must be approximated with the empirical symbol frequencies. The $k$-th order compression, however, stops being a reasonable model about the time when $\sigma^{k}$ becomes larger than $n$, where $\sigma$ and $n$ are the alphabet size and the string length, respectively. In particular, Gagie [18] showed that when $k \geq \log _{\sigma} n$, no compressed representation can achieve a worst-case space bound of $c \cdot n H_{k}+o(n \log \sigma)$ bits, regardless of the value of the constant $c$. This implies that $k$-th order entropy is a weak model when $k$ is large, i.e., when the goal is to capture long repetitions. Another way of proving this fact is to observe that, for any sufficiently long text $T$, symbol frequencies (after taking their context into account) in any power of $T$ (i.e., $T$ concatenated with itself) do not vary significantly [30, Lem. 2.6]. As a result, we have that $n H_{k}\left(T^{t}\right) \approx t \cdot n H_{k}(T)$ for any $t>1$ : entropy is not sensitive to very long repetitions.

This particular weakness of entropy compression generated, in the last couple of decades, a lot of interest in algorithms able to directly exploit text repetitiveness in order to beat the entropy lower bound on very repetitive texts. The main idea underlying these algorithms is to replace text substrings with references to a dictionary of strings, hence the name dictionary compressors. One effective compression strategy of this kind is to build a context-free grammar that

\section{KEYWORDS}


generates (only) the string. Such grammars (in Chomsky normal form) are known by the name of straight-line programs (SLP) [26]; an SLP is a set of rules of the kind $X \rightarrow A B$ or $X \rightarrow a$, where $X, A$, and $B$ are nonterminals and $a$ is a terminal. The string is obtained from the expansion of a single starting nonterminal $S$. If also rules of the form $X \rightarrow A^{\ell}$ are allowed, for any $\ell>2$, then the grammar is called run-length SLP (RLSLP) [36]. The problems of finding the smallest SLP - of size $g^{*}$ - and the smallest run-length SLP - of size $g_{r l}^{*}-$ are NP-hard [12, 23], but fast and effective approximation algorithms are known, e.g., LZ78 [46], LZW [44], Re-Pair [31], Bisection [27]. An even more powerful generalization of RLSLPs is represented by collage systems [25]: in this case, also rules of the form $X \rightarrow Y[l . . r]$ are allowed (i.e., $X$ expands to a substring of $Y$ ). We denote with $c$ the size of a generic collage system, and with $c^{*}$ the size of the smallest one. A related strategy, more powerful than grammar compression, is that of replacing repetitions with pointers to other locations in the string. The most powerful and general scheme falling into this category takes the name of pointer macro scheme [40, 41], and consists of a set of substring equalities that allow for unambiguously reconstructing the string. Finding the smallest such system - of size $b^{*}-$ is also NP-hard [22]. However, if we add the constraint of unidirectionality (i.e., text can only be copied from previous positions), then Lempel and Ziv in [32] showed that a greedy algorithm (LZ77) finds an optimal solution to the problem (we denote the size of the resulting parsing by $z$ ). Subsequent works showed that LZ77 can even be computed in linear time [14]. Other effective techniques to compress repetitive strings include the run-length Burrows-Wheeler transform [11] (RLBWT) and the compact directed acyclic word graph [10, 15] (CDAWG). With the first technique, we sort all circular string permutations in an $n \times n$ matrix; the BWT is the last column of this matrix. The BWT contains few equal-letter runs if the string is very repetitive, therefore run-length compression often significantly reduces the size of this string permutation [33]. The number $r$ of runs in the BWT is yet another good measure of repetitiveness. Finally, one can build a compact (that is, path compressed) automaton recognizing the string's suffixes, and indicate with $e$ the number of its edges. The size $e^{*}$ of the smallest such automaton - the CDAWG - also grows sublinearly with $n$ if the string is very repetitive [5]. Both RLBWT and CDAWG can be computed in linear time [1, 16, 35].

The promising results obtained in the field of dictionary compression have generated - in recent years - a lot of interest around the closely-related field of compressed computation, i.e., designing compressed data structures that efficiently support a particular set of queries on the text. The sizes of these data structures are bounded in terms of repetitiveness measures. As with text compression, the landscape of compressed data structures is extremely fragmented: different solutions exist for each compression scheme, and their space/query times are often not even comparable, due to the fact that many asymptotic relations between repetitiveness measures are still missing. See, for example, Gagie et al. [21] for a comprehensive overview of the state-of-the-art of dictionary-compressed full-text indexes (where considered queries are random access to text and counting/locating pattern occurrences). In this paper we consider data structures supporting random access queries (that is, efficient local decompression). Several data structures for this problem have been proposed in the literature for each distinct compression scheme. In Table 1 we report the best time-space trade-offs known to date (grouped by compression scheme). Extracting text from Lempel-Ziv compressed text is a notoriously difficult problem. No efficient solution is known within $O(z)$ space (they all require time proportional to the parse's height), although efficient queries can be supported by raising the space by a logarithmic factor $[6,8]$. Grammars, on the other hand, allow for more compact and time-efficient extraction strategies. Bille et al. [9] have been the first to show how to efficiently perform text extraction within $O(g)$ space. Their time bounds were later improved by Belazzougui et al. [2], who also showed how to slightly increase the space to $O\left(g \log ^{\epsilon} n \log (n / g)\right)$ while matching a lower bound holding on grammars [42]. Space-efficient text extraction from the run-length Burrows-Wheeler transform has been an open problem until recently. Standard solutions [33] required spending additional $O(n / s)$ space on top of the RLBWT in order to support extraction in a time proportional to $s$. In a recent publication, Gagie et al. [21] showed how to achieve near-optimal extraction time in the packed setting within $O(r \log (n / r))$ space. Belazzougui and Cunial [3] showed how to efficiently extract text from a CDAWG-compressed text. Their most recent work [4] shows, moreover, how to build a grammar of size $O(e)$ : this result implies that the solutions for grammarcompressed text can be used on the CDAWG. To conclude, no strategies for efficiently extracting text from general macro schemes and collage systems are known to date: the only solution we are aware of requires explicitly navigating the compressed structure, and can therefore take time proportional to the text's length in the worst case.

\subsection{Our Contributions}

At this point, it is natural to ask whether there exists a common (and simple) principle underlying the complex set of techniques constituting the fields of dictionary compression and compressedcomputation. In this paper, we answer (affirmatively) this question. Starting from the observation that string repetitiveness can be defined in terms of the cardinality of the set of distinct substrings, we introduce a very simple combinatorial object - the string attractor - capturing the complexity of this set. Formally, a string attractor is a subset of the string's positions such that all distinct substrings have an occurrence crossing at least one of the attractor's elements. Despite the simplicity of this definition, we show that dictionary compressors can be interpreted as algorithms approximating the smallest string attractor: they all induce (very naturally) string attractors whose sizes are bounded by their associated repetitiveness measures. We also provide reductions from string attractors to most dictionary compressors and use these reductions to derive their approximation rates with respect to the smallest string attractor. This yields our first efficient approximation algorithms computing the smallest string attractor, and allows us to uncover new relations between repetitiveness measures. For example, we show that $g^{*}, z \in O\left(c^{*} \log ^{2}\left(n / c^{*}\right)\right), c^{*} \in O\left(b^{*} \log \left(n / b^{*}\right)\right) \cap O(r \log (n / r))$, and $b^{*} \in O\left(c^{*} \log \left(n / c^{*}\right)\right)$.

Our reductions suggest that a solution (or a good approximation) to the problem of finding an attractor of minimum size could yield a better understanding of the concept of text repetitiveness and could 
help in designing better dictionary compressors. We approach the problem by first generalizing the notion of string attractor to that of $k$-attractor: a subset of the string's positions capturing all substrings of length at most $k$. We study the computational complexity of the $k$-attractor problem: to decide whether a text has a $k$-attractor of a given size. Using a reduction from $k$-set-cover, we show that the $k$-attractor problem is NP-complete for $k \geq 3$. In particular, this proves the NP-completeness of the original string attractor problem (i.e., the case $k=n$ ). Given the hardness of computing the smallest attractor, we focus on the problem of approximability. We show that the smallest $k$-attractor problem is APX-complete for constant $k$ by showing a $2 k$-approximation computable in linear time and a reduction from $k$-vertex-cover. We also use reductions to $k$-set-cover to provide $O(\log k)$-approximations computable in polynomial time. Our APX-completeness result implies that the smallest $k$-attractor problem has no PTAS unless $\mathrm{P}=\mathrm{NP}$. Using a reduction from 3-vertex-cover and explicit constants derived by Berman and Karpinski [7], we strengthen this result and show that, for every $\epsilon>0$ and every $k \geq 3$, it is NP-hard to approximate the smallest $k$-attractor within a factor of $11809 / 11808-\epsilon$.

We proceed by presenting an application of string attractors to the domain of compressed computation: we show that the simple property defining string attractors is sufficient to support random access in optimal time. We first extend a lower bound [42, Thm. 5] for random access on grammars to string attractors. Let $\gamma$ be the size of a string attractor of a length- $n$ string $T$ over an alphabet of size $\sigma$. The lower bound states that $\Omega(\log n / \log \log n)$ time is needed to access one random position within $O(\gamma$ polylog $n)$ space. Let $w$ be the memory word size (in bits). We present a data structure taking $O\left(\gamma \tau \log _{\tau}(n / \gamma)\right)$ words of space and supporting the extraction of any length- $\ell$ substring of $T$ in $O\left(\log _{\tau}(n / \gamma)+\ell \log \sigma / w\right)$ time, for any $\tau \geq 2$ fixed at construction time. For $\tau=\log ^{\epsilon} n$ (for any constant $\epsilon>0$ ) this query time matches the lower bound. Choosing $\tau=(n / \gamma)^{\epsilon}$, we obtain instead optimal time in the packed setting within $O\left(\gamma^{1-\epsilon} n^{\epsilon}\right)$ space. From our reductions, our solution is universal: given a dictionary-compressed text representation, we can induce a string attractor of the same size and build our structure on top of it. We note, as well, that the lower bound holds, in particular, on most compression schemes. As a result, our data structure is also optimal for SLPs, RLSLPs, collage systems, LZ77, and macro schemes. Tables 1 and 2 put our structure in the context of stateof-the-art solutions to the problem. Note that all existing solutions depend on a specific compression scheme.

\section{PRELIMINARIES}

Throughout the paper, we use the terms string and text interchangeably. The notion $T[i . . j], 1 \leq i \leq j \leq n$, denotes the substring of string $T \in \Sigma^{n}$ starting at position $i$ and ending at position $j$. We denote the alphabet size of string $T$ by $|\Sigma|=\sigma$.

The $L Z 77$ parsing $[32,45]$ of a string $T$ is a greedy, left-to-right parsing of $T$ into longest previous factors, where a longest previous factor at position $i$ is a pair $\left(p_{i}, \ell_{i}\right)$ such that, $p_{i}<i, T\left[p_{i} . . p_{i}+\ell_{i}-\right.$ $1]=T\left[i . . i+\ell_{i}-1\right]$, and $\ell_{i}$ is maximized. In this paper, we use the LZ77 variant where no overlaps between phrases and sources are allowed, i.e., we require that $p_{i}+\ell_{i}-1<i$. Elements of the parsing are called phrases. When $\ell_{i}>0$, the substring $T\left[p_{i} . . p_{i}+\ell_{i}-1\right]$ is
Table 1: Best trade-offs in the literature for extracting text from compressed representations.

\begin{tabular}{lcc}
\hline Structure & Space & Extract time \\
\hline [8, Lem. 5] & $O(z \log (n / z))$ & $O(\ell+\log (n / z))$ \\
[6, Thm. 2] & $O(z \log (n / z))$ & $O\left(\left(1+\ell / \log _{\sigma} n\right) \log (n / z)\right)$ \\
[2, Thm. 1] & $O(g)$ & $O\left(\ell / \log _{\sigma} n+\log n\right)$ \\
[2, Thm. 3] & $O\left(g \log ^{\epsilon} n \log \frac{n}{g}\right)$ & $O\left(\ell / \log _{\sigma} n+\frac{\log n}{\log \log n}\right)$ \\
[21, Thm. 4] & $O(r \log (n / r))$ & $O(\ell \log (\sigma) / w+\log (n / r))$ \\
[4, Thm. 7] & $O(e)$ & $O\left(\ell / \log _{\sigma} n+\log n\right)$ \\
\hline
\end{tabular}

Table 2: Some trade-offs achievable with our structure for different choices of $\tau$, in order of decreasing space and increasing time. Query time in the first row is optimal in the packed setting, while in the second row it is optimal within the resulting space due to a lower bound for random access on string attractors. To compare these bounds with those of Table 1 , just replace $\gamma$ with any of the measures $z, g, r$, or $e$ (possible by our reductions to string attractors).

\begin{tabular}{ccc}
\hline$\tau$ & Space & Extract time \\
\hline$(n / \gamma)^{\epsilon}$ & $O\left(\gamma^{1-\epsilon} n^{\epsilon}\right)$ & $O(\ell \log (\sigma) / w)$ \\
$\log ^{\epsilon} n$ & $O\left(\gamma \log ^{\epsilon} n \log (n / \gamma)\right)$ & $O\left(\ell \log (\sigma) / w+\frac{\log (n / \gamma)}{\log \log n}\right)$ \\
2 & $O(\gamma \log (n / \gamma))$ & $O(\ell \log (\sigma) / w+\log (n / \gamma))$ \\
\hline
\end{tabular}

called the source of phrase $T\left[i . . i+\ell_{i}-1\right]$. In other words, $T\left[i . . i+\ell_{i}-1\right]$ is the longest prefix of $T[i . . n]$ that has another occurrence not overlapping $T[i . . n]$ and $p_{i}<i$ is its starting position. The exception is when $\ell_{i}=0$, which happens iff $T[i]$ is the leftmost occurrence of a symbol in $T$. In this case we output $(T[i], 0)$ (to represent $T[i . . i]$ : a phrase of length 1) and the next phrase starts at position $i+1$. LZ77 parsing has been shown to be the smallest parsing of the string into phrases with sources that appear earlier in the text [45]. The parsing can be computed in $O(n)$ time for integer alphabets [14] and in $O(n \log \sigma)$ for general (ordered) alphabets [38]. The number of phrases in the LZ77 parsing of string $T$ is denoted by $z$.

A macro scheme [41] is a set of $b$ directives of two possible types:

(1) $T[i . . j] \leftarrow T\left[i^{\prime} . . j^{\prime}\right]$ (i.e., copy $T\left[i^{\prime} . . j^{\prime}\right]$ in $\left.T[i . . j]\right)$, or

(2) $T[i] \leftarrow c$, with $c \in \Sigma$ (i.e., assign character $c$ to $T[i]$ ), such that $T$ can be unambiguously decoded from the directives.

A bidirectional parse is a macro scheme where the left-hand sides of the directives induce a text factorization, i.e., they cover the whole $T$ and they do not overlap. Note that LZ77 is a particular case of a bidirectional parse (the optimal unidirectional one), and therefore it is also a macro scheme.

A collage system [25] is a set of $c$ rules of four possible types:

(1) $X \rightarrow a$ : nonterminal $X$ expands to a terminal $a$,

(2) $X \rightarrow A B$ : nonterminal $X$ expands to $A B$, with $A$ and $B$ nonterminals different from $X$,

(3) $X \rightarrow R^{\ell}$ : nonterminal $X$ expands to nonterminal $R \neq X$ repeated $\ell$ times,

(4) $X \rightarrow K[l . . r]$ : nonterminal $X$ expands to a substring of the expansion of nonterminal $K \neq X$. 
The text is the result of the expansion of a special starting nonterminal $S$. Moreover, we require that the collage system does not have cycles, i.e., the derivation tree of any nonterminal $X$ does not contain $X$ nor $X[l . . r]$ for some integers $l, r$ as an internal node. Collage systems generalize SLPs (where only rules 1 and 2 are allowed) and RLSLPs (where only rules 1, 2, and 3 are allowed). The height $h_{X}$ of a nonterminal $X$ is defined as follows. If $X$ expands to a terminal with rule 1 , then $h_{X}=1$. If $X$ expands to $A B$ with rule 2 , then $h_{X}=\max \left\{h_{A}, h_{B}\right\}+1$. If $X$ expands to $R^{\ell}$ with rule 3 , then $h_{X}=h_{R}+1$. If $X$ expands to $K[l . . r]$ with rule 4 , then $h_{X}=h_{K}+1$. The height of the collage system is the height of its starting nonterminal.

By $S A[1 . . n]$ we denote the suffix array [34] of $T,|T|=n$, defined as a permutation of the integers [1..n] such that $T[S A[1] . . n]<$ $T[S A[2] . . n] \prec \cdots \prec T[S A[n] . . n]$, where $\prec$ denotes the lexicographical ordering. For simplicity we assume that $T[n]=\$$, where $\$$ is a special symbol not occurring elsewhere in $T$ and lexicographically smaller than all other alphabet symbols. The Burrows-Wheeler Transform [11] BWT[1..n] is a permutation of the symbols in $T$ such that $B W T[i]=T[S A[i]-1]$ if $S A[i]>1$ and $\$$ otherwise. Equivalently, BWT can be obtained as follows: sort lexicographically all cyclic permutations of $T$ into a (conceptual) matrix $M \in \Sigma^{n \times n}$ and take its last column. Denote the first and last column of $M$ by $F$ and $L$, respectively. The key property of $M$ is the $L F$ mapping: the $i$-th occurrence of any character $c$ in column $L$ corresponds to the $i$-th occurrence of any character $c$ in column $F$ (i.e., they represent the same position in the text). With $L F[i], i \in[1, n]$ we denote the LF mapping applied on position $i$ in the $L$ column. It is easy to show that $L F[i]=C[L[i]]+\operatorname{rank}_{L[i]}(L, i)$, where $C[c]=|\{i \in[1, n] \mid L[i]<c\}|$ and $\operatorname{rank}_{c}(L, i)$ is the number of occurrences of $c$ in $L[1 . . i-1]$.

On compressible texts, BWT exhibits some remarkable properties that allow the boosting of compression. In particular, it can be shown [33] that repetitions in $T$ generate equal-letter runs in BWT. We can efficiently represent this transform as the list of pairs

$$
R L B W T=\left\langle\lambda_{i}, c_{i}\right\rangle_{i=1, \ldots, r},
$$

where $\lambda_{i}>0$ is the length of the $i$-th maximal run, and $c_{i} \in \Sigma$. Equivalently, $R L B W T$ is the shortest list of pairs $\left\langle\lambda_{i}, c_{i}\right\rangle_{i=1, \ldots, r}$ satisfying $B W T=c_{1}^{\lambda_{1}} c_{2}^{\lambda_{2}} \ldots c_{r}^{\lambda_{r}}$.

The compact directed acyclic word graph [10, 15] (CDAWG for short) is the minimum path-compressed graph (i.e., unary paths are collapsed into one path) with one source node $s$ and one sink node $f$ such that all $T$ 's suffixes can be read on a path starting from the source. The CDAWG can be built in linear time by minimization of the suffix tree [43] of $T$ : collapse all leaves in one single node, and proceed bottom-up until no more nodes of the suffix tree can be collapsed. The CDAWG can be regarded as an automaton recognizing all T's substrings: make $s$ the initial automaton's state and all other nodes (implicit and explicit) final.

\section{STRING ATTRACTORS}

A string $T[1 . . n]$ is considered to be repetitive when the cardinality of the set $S U B_{T}=\{T[i . . j]|1 \leq i \leq j \leq| T \mid\}$ of its distinct substrings is much smaller than the maximum number of distinct substrings that could appear in a string of the same length on the same alphabet.
Note that $T$ can be viewed as a compact representation of $S U B_{T}$. This observation suggests a simple way of capturing the degree of repetitiveness of $T$, i.e., the cardinality of $S U B_{T}$. We can define a function $\phi: S U B_{T} \rightarrow \Gamma \subseteq[1, n]$ satisfying the following property: each $s \in S U B_{T}$ has an occurrence crossing position $\phi(s)$ in $T$. Note that such a function is not necessarily unique. The codomain $\Gamma$ of $\phi$ is the object of study of this paper. We call this set a string attractor:

Definition 3.1. A string attractor of a string $T \in \Sigma^{n}$ is a set of $\gamma$ positions $\Gamma=\left\{j_{1}, \ldots, j_{\gamma}\right\}$ such that every substring $T[i . . j]$ has an occurrence $T\left[i^{\prime} . . j^{\prime}\right]=T[i . . j]$ with $j_{k} \in\left[i^{\prime}, j^{\prime}\right]$, for some $j_{k} \in \Gamma$.

Example 3.2. Note that $\{1,2, . ., n\}$ is always a string attractor (the largest one) for any string. Note also that this is the only possible string attractor for a string composed of $n$ distinct characters (e.g., a permutation).

Example 3.3. Consider the following string $T$, where we underlined the positions of a smallest string attractor $\Gamma^{*}=\{4,7,11,12\}$ of $T$.

\section{CDABCCDABCCA}

To see that $\Gamma^{*}$ is a valid attractor, note that every substring between attractor's positions has an occurrence crossing some attractor's position (these substrings are $A, B, C, D, C D, D A, C C, A B, B C, C D A, A B C$ ). The remaining substrings cross an attractor's position by definition. To see that $\Gamma^{*}$ is of minimum size, note that the alphabet size is $\sigma=4=\left|\Gamma^{*}\right|$, and any attractor $\Gamma$ must satisfy $|\Gamma| \geq \sigma$.

\subsection{Reductions from Dictionary Compressors}

In this section we show that dictionary compressors induce string attractors whose sizes are bounded by their associated repetitiveness measures.

Since SLPs and RLSLPs are particular cases of collage systems, we only need to show a reduction from collage systems to string attractors to capture these three classes of dictionary compressors. We start with the following auxiliary lemma.

LemmA 3.4. Let $C=\left\{X_{i} \rightarrow a_{i}, i=1, \ldots, c^{\prime}\right\} \cup\left\{Y_{i} \rightarrow A_{i} B_{i}, i=\right.$ $\left.1, \ldots, c^{\prime \prime}\right\} \cup\left\{Z_{i} \rightarrow R_{i}^{\ell_{i}}, \ell_{i}>2, i=1, \ldots, c^{\prime \prime \prime}\right\} \cup\left\{W_{i} \rightarrow K_{i}\left[l_{i} . . r_{i}\right], i=\right.$ $\left.1, \ldots, c^{\prime \prime \prime \prime}\right\}$ be a collage system with starting nonterminal $S$ generating string $T$. For any substring $T[i . . j]$ one of the following is true:

(1) $i=j$ and $T[i]=a_{k}$, for some $1 \leq k \leq c^{\prime}$, or

(2) there exists a rule $Y_{k} \rightarrow A_{k} B_{k}$ such that $T[i . . j]$ is composed of a non-empty suffix of the expansion of $A_{k}$ followed by a non-empty prefix of the expansion of $B_{k}$, or

(3) there exists a rule $Z_{k} \rightarrow R_{k}^{\ell_{k}}$ such that $T[i . . j]$ is composed of a non-empty suffix of the expansion of $R_{k}$ followed by $a$ non-empty prefix of the expansion of $R_{k}^{\ell_{k}-1}$.

Proof. Consider any substring $T[i . . j]$ generated by expanding the start rule $S$. The proof is by induction on the height $h$ of $S$.

For $h=1$, the start rule $S$ must expand to a single symbol and hence case (1) holds. Consider a collage system of height $h>1$, and let $S$ be its start symbol. Then, $S$ has one of the following forms:

(1) $S$ expands as $S \rightarrow A B$, or

(2) $S$ expands as $S \rightarrow R^{\ell}$, or

(3) $S$ expands as $S \rightarrow K[l . . r]$, 
where $A, B, R$, and $K$ are all nonterminals of height $h-1$.

In case (1), either $T[i . . j]$ is fully contained in the expansion of $A$, or it is fully contained in the expansion of $B$, or it is formed by a non-empty suffix of the expansion of $A$ followed by a non-empty prefix of the expansion of $B$. In the first two cases, our claim is true by inductive hypothesis on the collage systems with start symbols $A$ or $B$. In the third case, our claim is true by definition.

In case (2), either $T[i . . j]$ is fully contained in the expansion of $R$, or it is formed by a non-empty suffix of the expansion of $R^{\ell_{1}}$ followed by a non-empty prefix of the expansion of $R^{\ell_{2}}$, for some $\ell_{1}, \ell_{2}>0$ such that $\ell_{1}+\ell_{2}=\ell$. In the former case, our claim is true by inductive hypothesis. In the latter case, $T[i . . j]$ can be written as a suffix of $R$ followed by a concatenation of $k \geq 0$ copies of $R$ followed by a prefix of $R$, i.e., $T[i . . j]=R[l . .|R|] R^{k} R[1 . . r]$ for some $1 \leq l \leq$ $|R|, k \geq 0$, and $1 \leq r \leq|R|$. Then, $T[i . . j]$ has also an occurrence crossing $R$ and $R^{\ell-1}: T[i . . j]=R[l . .|R|] R^{\ell-1}[1 . .(j-i)-(|R|-l)]$.

In case (3), since $T[i . . j]$ is a substring of the expansion of $S$, then it is also a substring of the expansion of $K$. Since the height of $K$ is $h-1$, we apply an inductive hypothesis with start symbol $K$.

The above lemma leads to our first reduction:

Theorem 3.5. Let $C$ be a collage system of size $c$ generating $T$. Then, $T$ has an attractor of size at most $c$.

Proof. Let $C=\left\{X_{i} \rightarrow a_{i}, i=1, \ldots, c^{\prime}\right\} \cup\left\{Y_{i} \rightarrow A_{i} B_{i}, i=\right.$ $\left.1, \ldots, c^{\prime \prime}\right\} \cup\left\{Z_{i} \rightarrow R_{i}^{\ell_{i}}, \ell_{i}>2, i=1, \ldots, c^{\prime \prime \prime}\right\} \cup\left\{W_{i} \rightarrow K_{i}\left[l_{i} . . r_{i}\right], i=\right.$ $\left.1, \ldots, c^{\prime \prime \prime \prime}\right\}$ be a collage system of size $c=c^{\prime}+c^{\prime \prime}+c^{\prime \prime \prime}+c^{\prime \prime \prime \prime}$ generating string $T$.

Start with an empty string attractor $\Gamma_{C}=\emptyset$ and repeat the following for $k=1, \ldots, c^{\prime}$. Choose any of the occurrences $T[i]$ of the expansion $a_{k}$ of $X_{k}$ in $T$ and insert $i$ in $\Gamma_{C}$. Next, for $k=$ $1, \ldots, c^{\prime \prime}$, choose any of the occurrences $T[i . . j]$ of the expansion of $Y_{k}$. By the production $Y_{k} \rightarrow A_{k} B_{k}, T[i . . j]$ can be factored as $T[i . . j]=T\left[i . . i^{\prime}\right] T\left[i^{\prime}+1 . . j\right]$, where $T\left[i . . i^{\prime}\right]$ and $T\left[i^{\prime}+1 . . j\right]$ are expansions of $A_{k}$ and $B_{k}$, respectively. Insert position $i^{\prime}$ in $\Gamma_{C}$. Finally, for $k=1, \ldots, c^{\prime \prime \prime}$, choose any of the occurrences $T[i . . j]$ of the expansion of $Z_{k}$ in $T$. By the production $Z_{k} \rightarrow R_{k}^{\ell_{k}}, T[i . . j]$ can be factored as $T[i . . j]=T\left[i . . i^{\prime}\right] T\left[i^{\prime}+1 . . j\right]$, where $T\left[i . . i^{\prime}\right]$ and $T\left[i^{\prime}+1 . . j\right]$ are expansions of $R_{k}$ and $R_{k}^{\ell_{k}-1}$. Insert position $i^{\prime}$ in $\Gamma_{C}$.

Clearly the size of $\Gamma_{C}$ is at most $c$. To see that $\Gamma_{C}$ is a valid attractor, consider any substring $T[i . . j]$ of $T$. By Lemma 3.4, either $i=j$ (and, by construction, $\Gamma_{C}$ contains a position of some occurrence of $a_{k}$ such that $T[i]=a_{k}$ and $X_{k} \rightarrow a_{k}$ is one of the rules in $C$ ), or $T[i . . j]$ spans the expansion of some $A_{k} \mid B_{k}$ or $R_{k} \mid R_{k}^{\ell_{k}-1}$ (with the crossing point shown). From the construction of $\Gamma_{C}$, such expansion has an occurrence in $T$ containing an element in $\Gamma_{C}$ right before the crossing point. Thus, $T[i . . j]$ has an occurrence $T\left[i^{\prime} . . j^{\prime}\right]$ containing a position from $\Gamma_{C}$.

We now show an analogous result for macro schemes.

THEOREM 3.6. Let $M$ be a macro scheme of size $b$ of T. Then, $T$ has an attractor of size at most $2 b$.

Proof. Let $T\left[i_{k_{1}} . . j_{k_{1}}\right] \leftarrow T\left[i_{k_{1}}^{\prime} . . j_{k_{1}}^{\prime}\right], T\left[q_{k_{2}}\right] \leftarrow c_{k_{2}}$, with $1 \leq$ $k_{1} \leq b_{1}, 1 \leq k_{2} \leq b_{2}$, and $b=b_{1}+b_{2}$ be the $b$ directives of our macro scheme MS. We claim that $\Gamma_{M S}=\left\{i_{1}, \ldots, i_{b_{1}}, j_{1}, \ldots, j_{b_{1}}, q_{1}, \ldots, q_{b_{2}}\right\}$ is a valid string attractor for $T$.
Let $T[i . . j]$ be any substring. All we need to show is that $T[i . . j]$ has a primary occurrence, i.e., an occurrence containing one of the positions $i_{k_{1}}, j_{k_{1}}$, or $q_{k_{2}}$. Let $s_{1}=i$ and $t_{1}=j$. Consider all possible chains of copies (following the macro scheme directives) $T\left[s_{1} . . t_{1}\right] \leftarrow T\left[s_{2} . . t_{2}\right] \leftarrow T\left[s_{3} . . t_{3}\right] \leftarrow \ldots$ that either end in some primary occurrence $T\left[s_{k} . . t_{k}\right]$ or are infinite (note that there could exist multiple chains of this kind since the left-hand side terms of some macro scheme's directives could overlap). Our goal is to show that there must exist at least one finite such chain, i.e., that ends in a primary occurrence. Pick any $s_{1} \leq p_{1} \leq t_{1}$. Since ours is a valid macro scheme, then $T\left[p_{1}\right]$ can be retrieved from the scheme, i.e., the directives induce a finite chain of copies $T\left[p_{1}\right] \leftarrow$ $\cdots \leftarrow T\left[p_{k^{\prime}}\right] \leftarrow c$, for some $k^{\prime}$, such that $T\left[p_{k^{\prime}}\right] \leftarrow c$ is one of the macro scheme's directives. We now show how to build a finite chain of copies $T\left[s_{1} . . t_{1}\right] \leftarrow T\left[s_{2} . . t_{2}\right] \leftarrow \cdots \leftarrow T\left[s_{k} . . t_{k}\right]$ ending in a primary occurrence $T\left[s_{k} . . t_{k}\right]$ of $T\left[s_{1} . . t_{1}\right]$, with $k \leq k^{\prime}$. By definition, the assignment $T\left[p_{1}\right] \leftarrow T\left[p_{2}\right]$ comes from some macro scheme's directive $T\left[l_{1} . . r_{1}\right] \leftarrow T\left[l_{2} . . r_{2}\right]$ such that $p_{1} \in\left[l_{1}, r_{1}\right]$ and $p_{1}-l_{1}=$ $p_{2}-l_{2}$ (if there are multiple directives of this kind, pick any of them). If either $l_{1} \in\left[s_{1}, t_{1}\right]$ or $r_{1} \in\left[s_{1}, t_{1}\right]$, then $T\left[s_{1} . . t_{1}\right]$ is a primary occurrence and we are done. Otherwise, we set $s_{2}=l_{2}+\left(i-l_{1}\right)$ and $t_{2}=l_{2}+\left(j-l_{1}\right)$. By this definition, we have that $T\left[s_{1} . . t_{1}\right]=T\left[s_{2} . . t_{2}\right]$ and $p_{2} \in\left[s_{2}, t_{2}\right]$, therefore we can extend our chain to $T\left[s_{1} . . t_{1}\right] \leftarrow$ $T\left[s_{2} . . t_{2}\right]$. It is clear that the reasoning can be repeated, yielding that either $T\left[s_{2} . . t_{2}\right]$ is a primary occurrence or our chain can be extended to $T\left[s_{1} . . t_{1}\right] \leftarrow T\left[s_{2} . . t_{2}\right] \leftarrow T\left[s_{3} . . t_{3}\right]$ for some substring $T\left[s_{3} . . t_{3}\right]$ such that $p_{3} \in\left[s_{3}, t_{3}\right]$. We repeat the construction for $p_{4}, p_{5}, \ldots$ until either (i) we end up in a chain $T[i . . j] \leftarrow \cdots \leftarrow T\left[s_{k} . . t_{k}\right]$, with $k<k^{\prime}$, ending in a primary occurrence $T\left[s_{k} . . k_{k}\right]$ of $T\left[s_{1} . . t_{1}\right]$, or (ii) we obtain a chain $T\left[s_{1} . . t_{1}\right] \leftarrow \cdots \leftarrow T\left[s_{k^{\prime}} . . t_{k^{\prime}}\right]$ such that $p_{k^{\prime}} \in\left[s_{k^{\prime}}, t_{k^{\prime}}\right]$ (i.e., we consume all the $p_{1}, \ldots, p_{k^{\prime}}$ ). In case (ii), note that $T\left[p_{k^{\prime}}\right] \leftarrow c$ is one of the macro scheme's directives, therefore $T\left[s_{k^{\prime}} . . t_{k^{\prime}}\right]$ is a primary occurrence of $T\left[s_{1} . . t_{1}\right]$.

The above theorem implies that LZ77 induces a string attractor of size at most $2 z$. We can achieve a better bound by exploiting the so-called primary occurrence property of LZ77:

LEMмA 3.7. Let $z$ be the number of factors of the Lempel-Ziv factorization of a string $T$. Then, $T$ has an attractor of size $z$.

Proof. We insert in $\Gamma_{L Z 77}$ all positions at the end of a phrase. It is easy to see [30] that every text substring has an occurrence crossing a phrase boundary (these occurrences are called primary), therefore we obtain that $\Gamma_{L Z 77}$ is a valid attractor for $T$.

Kosaraju and Manzini [29] showed that LZ77 is coarsely optimal, i.e., its compression ratio differs from the $k$-th order empirical entropy by a quantity tending to zero as the text length increases. From Lemma 3.7, we can therefore give an upper bound to the size of the smallest attractor in terms of $k$-th order empirical entropy. ${ }^{1}$

COROLlaRy 3.8. Let $\gamma^{*}$ be the size of the smallest attractor for a string $T \in \Sigma^{n}$, and $H_{k}$ denote the $k$-th order empirical entropy of $T$. Then, $\gamma^{*} \log n \leq n H_{k}+o(n \log \sigma)$ for $k \in o\left(\log _{\sigma} n\right)$.

${ }^{1}$ Note that [29] assumes a version of LZ77 that allows phrases to overlap their sources. It is easy to check that Lemma 3.7 holds also for this variant. 
The run-length Burrows-Wheeler transform seems a completely different paradigm for compressing repetitive strings: while grammars and macro schemes explicitly copy portions of the text to other locations, with the RLBWT we build a string permutation by concatenating characters preceding lexicographically-sorted suffixes, and then run-length compress it. This strategy is motivated by the fact that equal substrings are often preceded by the same character, therefore the BWT contains long runs of the same letter if the string is repetitive [33]. We obtain:

THEOREM 3.9. Let $r$ be the number of equal-letter runs in the Burrows-Wheeler transform of T. Then, $T$ has an attractor of size $r$.

Proof. Let $n=|T|$. Denote the BWT of $T$ by $L$ and consider the process of inverting the BWT to obtain $T$. The inversion algorithm is based on the observation that $T[n-k]=L\left[L F^{k}\left[p_{0}\right]\right]$ for $k \in[0, n-1]$, where $p_{0}$ is the position of $T[n]$ in $L$. From the formula for $L F$ it is easy to see that if two positions $i, j$ belong to the same equal-letter run in $L$ then $L F[j]=L F[i]+(j-i)$. Let $\Gamma_{B W T}=\left\{n-k \mid L F^{k}\left[p_{0}\right]=\right.$ 1 or $\left.L\left[L F^{k}\left[p_{0}\right]-1\right] \neq L\left[L F^{k}\left[p_{0}\right]\right]\right\}$, i.e., $\Gamma_{B W T}$ is the set of positions $i$ in $T$ such that if the symbol in $L$ corresponding to $T[i]$ is $L[j]$ then $j$ is the beginning of run in $L$ (alternatively, we can define $\Gamma_{B W T}$ as the set of positions at the end of BWT runs).

To show that $\Gamma_{B W T}$ is an attractor of $T$, consider any substring $T[i . . j]$ of $T$. We show that there exists an occurrence of $T[i . . j]$ in $T$ that contains at least one position from $\Gamma_{B W T}$. Let $p=L F^{n-j}\left[p_{0}\right]$, i.e., $L[p]$ is the symbol in $L$ corresponding to $T[j]$. Denote $\ell=$ $j-i+1$ and let $\left[i_{0}, j_{0}\right],\left[i_{1}, j_{1}\right], \ldots,\left[i_{\ell-1}, j_{\ell-1}\right]$ be the sequence of runs visited when the BWT inversion algorithm computes, from right to left, $T[i . . j]$, i.e., $L\left[i_{t} . . j_{t}\right]$ is the BWT-run containing $L\left[L F^{t}[p]\right]$, $t \in\{0, \ldots, \ell-1\}$. Let $b=\operatorname{argmin}\left\{L F^{t}[p]-i_{t} \mid t \in[0, \ell-1]\right\}$. Further, let $\Delta=L F^{b}[p]-i_{b}$ and let $p^{\prime}=p-\Delta$. By definition of $b$ and from the above property of $L F$ for two positions inside the same run we have that $L\left[L F^{t}\left[p^{\prime}\right]\right]=L\left[L F^{t}[p]\right]$ for $t \in[0, \ell-1]$. This implies that if we let $j^{\prime}$ be such that $p^{\prime}=L F^{n-j^{\prime}}\left[p_{0}\right]$ (i.e., $j^{\prime}$ is such that $T\left[j^{\prime}\right]$ corresponds to $\left.L\left[p^{\prime}\right]\right)$ then $T[i . . j]=T\left[i^{\prime} . . j^{\prime}\right]$ for $i^{\prime}:=j^{\prime}-\ell+1$. However, since by definition of $b, L\left[L F^{b}\left[p^{\prime}\right]\right]$ (corresponding to $T\left[j^{\prime}-b\right]$ ) is at the beginning of run in $L, T\left[i^{\prime} . . j^{\prime}\right]$ contains a position from $\Gamma_{B W T}$.

Finally, an analogous theorem holds for automata recognizing the string's suffixes:

THEOREM 3.10. Let e be the number of edges of a compact automaton $\mathcal{A}$ recognizing all (and only the) suffixes of a string $T$. Then, $T$ has an attractor of size e.

Proof. We call root the starting state of $\mathcal{A}$. Start with empty attractor $\Gamma_{\mathcal{A}}$. For every edge $(u, v)$ of $\mathcal{A}$, do the following. Let $T[i . . j]$ be any occurrence of the substring read from the root of $\mathcal{A}$ to the first character in the label of $(u, v)$. We insert $j$ in $\Gamma_{\mathcal{A}}$.

To see that $\Gamma_{\mathcal{A}}$ is a valid string attractor of size $e$, consider any substring $T[i . . j]$. By definition of $\mathcal{A}, T[i . . j]$ defines a path from the root to some node $u$, plus a prefix of the label (possibly, all the characters of the label) of an edge $(u, v)$ originating from $u$. Let $T[i . . k], k \leq j$, be the string read from the root to $u$, plus the first character in the label of $(u, v)$. Then, by definition of $\Gamma_{\mathcal{A}}$ there is an occurrence $T\left[i^{\prime} . . k^{\prime}\right]=T[i . . k]$ such that $k^{\prime} \in \Gamma_{\mathcal{A}}$. Since the remaining (possibly empty) suffix $T[k+1 . . j]$ of $T[i . . j]$ ends in the middle of an edge, every occurrence of $T[i . . k]$ is followed by $T[k+1 . . j]$, i.e., $T\left[i^{\prime} . . i^{\prime}+(j-i)\right]$ is an occurrence of $T[i . . j]$ crossing the attractor's element $k^{\prime}$.

\subsection{Reductions to Dictionary Compressors}

In this section we show reductions from string attractors to dictionary compressors. Combined with the results of the previous section, this will imply that dictionary compressors can be interpreted as approximation algorithms for the smallest string attractor. The next property follows easily from Definition 3.1 and will be used in the proofs of the following theorems.

Lemma 3.11. Any superset of a string attractor is also a string attractor.

We now show that we can derive a bidirectional parse from a string attractor.

THeOREM 3.12. Given a string $T \in \Sigma^{n}$ and a string attractor $\Gamma$ of size $\gamma$ for $T$, we can build a bidirectional parse (and therefore a macro scheme) for $T$ of size $O(\gamma \log (n / \gamma))$.

Proof. We add $\gamma$ equally-spaced attractor's elements following Lemma 3.11. We define phrases of the parse around attractor's elements in a "concentric exponential fashion", as follows. Characters on attractor's positions are explicitly stored. Let $i_{1}<i_{2}$ be two consecutive attractor's elements. Let $m=\left\lfloor\left(i_{1}+i_{2}\right) / 2\right\rfloor$ be the middle position between them. We create a phrase of length 1 in position $i_{1}+1$, followed by a phrase of length 2 , followed by a phrase of length 4, and so on. The last phrase is truncated at position $m$. We do the same (but right-to-left) for position $i_{2}$ except the last phrase is truncated at position $m+1$. For the phrases' sources, we use any of their occurrences crossing an attractor's element (possible by definition of $\Gamma$ ).

Suppose we are to extract $T[i]$, and $i$ is inside a phrase of length $\leq 2^{e}$, for some $e$. Let $i^{\prime}$ be the position from where $T[i]$ is copied according to our bidirectional parse. By the way we defined the scheme, it is not hard to see that $i^{\prime}$ is either an explicitly stored character or lies inside a phrase of length ${ }^{2} \leq 2^{e-1}$. Repeating the reasoning, we will ultimately "fall" on an explicitly stored character. Since attractor's elements are at a distance of at most $n / \gamma$ from each other, both the parse height and the number of phrases we introduce per attractor's element $\operatorname{are} O(\log (n / \gamma))$.

The particular recursive structure of the macro scheme of Theorem 3.12 can be exploited to induce a collage system of the same size. We state this result in the following theorem.

THEOREM 3.13. Given a string $T \in \Sigma^{n}$ and a string attractor $\Gamma$ of size $\gamma$ for $T$, we can build a collage system for $T$ of size $O(\gamma \log (n / \gamma))$.

Proof. We first build the bidirectional parse of Theorem 3.12, with $O(\gamma \log (n / \gamma))$ phrases of length at most $n / \gamma$ each. We maintain the following invariant: every maximal substring $T[i . . j]$ covered by processed phrases is collapsed into a single nonterminal $Y$.

We will process phrases in order of increasing length. The idea is to map a phrase on its source and copy the collage system of the

\footnotetext{
${ }^{2}$ To see this, note that $2^{e}=1+2^{0}+2^{1}+2^{2}+\cdots+2^{e-1}$ : these are the lengths of phrases following (and preceding) attractor's elements (included). In the worst case, position $i^{\prime}$ falls inside the longest such phrase (of length $2^{e-1}$ ).
} 
source introducing only a constant number of new nonterminals. By the bidirectional parse's definition, the source of any phrase $T[i . . j]$ overlaps only phrases shorter than $j-i+1$ characters. Since we process phrases in order of increasing length, phrases overlapping the source have already been processed and therefore $T[i . . j]$ is a substring of the expansion of some existing nonterminal $K$.

We start by parsing each maximal substring $T[i . . j]$ containing only phrases of length 1 into arbitrary blocks of length 2 or 3 . We create a constant number of new nonterminals per block (one for blocks of length two, and two for blocks of length three). Note that, by the way the parse is defined, this is always possible (since $j-i+1 \geq 2$ always holds). We repeat this process recursively grouping nonterminals at level $k \geq 0$ to form new nonterminals at level $k+1-$ until $T[i . . j]$ is collapsed into a single nonterminal. Our invariant now holds for the base case, i.e., for phrases of length $t=1$ : each maximal substring containing only phrases of length $\leq t$ is collapsed into a single nonterminal.

We now proceed with phrases of length $\geq 2$, in order of increasing length. Let $T[i . . j]$ be a phrase to be processed, with source at $T\left[i^{\prime} . . j^{\prime}\right]$. By definition of the parse, $T\left[i^{\prime} . . j^{\prime}\right]$ overlaps only phrases of length at most $j-i$ and, by inductive hypothesis, these phrases have already been processed. It follows that $T\left[i^{\prime} . . j^{\prime}\right]$ is equal to a substring $K\left[i^{\prime \prime} . . j^{\prime \prime}\right]$ of the expansion of some existing nonterminal $K$. At this point, it is sufficient to add a new rule $W \rightarrow K\left[i^{\prime \prime} . . j^{\prime \prime}\right]$ generating our phrase $T[i . . j]$. Since we process phrases in order of increasing length, $W$ is either followed $\left(W X_{1}\right)$, preceded $\left(X_{1} W\right)$, or in the middle $\left(X_{1} W X_{2}\right)$ of one or two nonterminals $X_{1}, X_{2}$ expanding to a maximal substring containing adjacent processed phrases. We introduce at most two new rules of the form $Y \rightarrow A B$ to merge these nonterminals into a single nonterminal, so that our invariant is still valid. Since we introduce a constant number of new nonterminals per phrase, the resulting collage system has $O(\gamma \log (n / \gamma))$ rules.

A similar proof can be used to derive a (larger) straight-line program.

THEOREM 3.14. Given a string $T \in \Sigma^{n}$ and a string attractor $\Gamma$ of size $\gamma$ for $T$, we can build an SLP for $T$ of size $O\left(\gamma \log ^{2}(n / \gamma)\right)$.

Proof. This proof follows that for Theorem 3.13 (but is slightly more complicated since we cannot use rules of the form $W \rightarrow$ $K[l . . r])$. We will first show a simpler construction achieving an SLP of size $O(\gamma \log (n / \gamma) \log (n))$ and then show how to refine it to achieve size $O\left(\gamma \log ^{2}(n / \gamma)\right)$.

For the purpose of the proof we modify the definition of SLP to allow also for the rules of the form $A \rightarrow X Y Z, A \rightarrow a b$, and $A \rightarrow a b c$ where $\{X, Y, Z\}$ are nonterminals and $\{a, b, c\}$ are terminals. It is easy to see that, if needed, the final SLP can be turned into a standard SLP without asymptotically affecting its size and height.

For any nonterminal, by level we mean the height of its parsetree. This is motivated by the fact that at any point during the construction, any nonterminal at level $k$ will have all its children at level exactly $k-1$. Furthermore, once a nonterminal is created, it is never changed or deleted. Levels of nonterminals, in particular, will thus not change during construction.

We start by building the bidirectional parse of Theorem 3.12, with $O(\gamma \log (n / \gamma))$ phrases of length at most $n / \gamma$ each. We will process phrases in order of increasing length. The main idea is to map a phrase on its source and copy the source's parse into nonterminals, introducing new nonterminals at the borders if needed. By the bidirectional parse's definition, the source of any phrase $T[i . . j]$ overlaps only phrases shorter than $j-i+1$ characters. Since we process phrases in order of increasing length, phrases overlapping the source have already been processed and therefore their parse into nonterminals is well-defined. We will maintain the following invariant: once we finish the processing of a phrase with the source $T\left[i^{\prime} . . j^{\prime}\right]$, the phrase will be represented by a single nonterminal $Y$ (expanding to $T\left[i^{\prime} . . j^{\prime}\right]$ ).

In the first version of our construction we will also maintain the following invariant: every maximal substring $T[i . . j]$ covered by processed phrases is collapsed into a single nonterminal $X$. Hence, whenever the processing of some phrase is finished and its source is an expansion of some nonterminal $Y$ we have to merge it with at most two adjacent nonterminals representing contiguous processed phrases to keep our invariant true. It is clear that, once all phrases have been processed, the entire string is collapsed into a single nonterminal $S$. We now show how to process a phrase and analyze the number of introduced nonterminals.

We start by parsing each maximal substring $T[i . . j]$ containing only phrases of length 1 into arbitrary blocks of length 2 or 3 . We create a new nonterminal for every block. We then repeat this process recursively - grouping nonterminals at level $k \geq 0$ to form new nonterminals at level $k+1-$ until $T[i . . j]$ is collapsed into a single nonterminal. Our invariant now holds for the base case $t=1$ : each maximal substring containing only phrases of length $\leq t$ is collapsed into a single nonterminal.

We now proceed with phrases of length $\geq 2$, in order of increasing length. Let $T[i . . j]$ be a phrase to be processed, with source at $T\left[i^{\prime} . . j^{\prime}\right]$. By definition of the parse, $T\left[i^{\prime} . . j^{\prime}\right]$ overlaps only phrases of length at most $j-i$ and, by inductive hypothesis, these phrases have already been processed. We group characters of $T[i . . j]$ in blocks of length 2 or 3 copying the parse of $T\left[i^{\prime} . . j^{\prime}\right]$ at level 0 . Note that this might not be possible for the borders of length 1 or 2 of $T[i . . j]$ : this is the case if the block containing $T\left[i^{\prime}\right]$ starts before position $i^{\prime}$ (symmetric for $T\left[j^{\prime}\right]$ ). In this case, we create $O(1)$ new nonterminals as follows. If $T\left[i^{\prime}-1, i^{\prime}, i^{\prime}+1\right]$ form a block, then we group $T[i, i+1]$ in a block of length 2 and collapse it into a new nonterminal at level 1 . If, on the other hand, $T\left[i^{\prime}-1, i^{\prime}\right]$ form a block, we consider two sub-cases. If $T\left[i^{\prime}+1, i^{\prime}+2\right]$ form a block, then we create the block to $T[i, i+1, i+2]$ and collapse it into a new nonterminal at level 1 . If $T\left[i^{\prime}+1, i^{\prime}+2, i^{\prime}+3\right]$ form a block, then we create the two blocks $T[i, i+1]$ and $T[i+2, i+3]$ and collapse them into 2 new nonterminals at level 1 . Finally, the case when $T\left[i^{\prime}-2, i^{\prime}-1, i^{\prime}\right]$ form a block is handled identically to the previous case. We repeat this process for the nonterminals at level $k \geq 1$ that were copied from $T\left[i^{\prime} . . j^{\prime}\right]$, grouping them in blocks of length 2 or 3 according to the source and creating $O(1)$ new nonterminals at level $k+1$ to cover the borders. After $O(\log (n / \gamma))$ levels, $T[i . . j]$ is collapsed into a single nonterminal. Since we create $O(1)$ new nonterminals per level, overall we introduce $O(\log (n / \gamma))$ new nonterminals.

At this point, let $Y$ be the nonterminal just created that expands to $T[i . . j]$. Since we process phrases in order of increasing length, $Y$ is either followed $(Y X)$, preceded $(X Y)$, or in the middle $\left(X_{1} Y X_{2}\right)$ of 
one or two nonterminals expanding to a maximal substring containing contiguous processed phrases. We now show how to collapse these two or three nonterminals in order to maintain our invariant, at the same time satisfying the property that nonterminals at level $k$ expand to two or three nonterminals at level $k-1$. We show the procedure in the case where $Y$ is preceded by a nonterminal $X$, i.e., we want to collapse $X Y$ into a single nonterminal. The other two cases can then easily be derived using the same technique. Let $k_{X}$ and $k_{Y}$ be the levels of $X$ and $Y$. If $k_{X}=k_{Y}$, then we just create a new nonterminal $W \rightarrow X Y$ and we are done. Assume first that $k_{X} \leq k_{Y}$. Let $Y_{1} \ldots Y_{t}$, with $t \geq 2$, be the sequence of nonterminals that are the expansion of $Y$ at level $k_{X}$. Our goal is to collapse the sequence $X Y_{1} \ldots Y_{t}$ into a single nonterminal. We will show that this is possible while introducing at most $O(\log (n / \gamma))$ new nonterminals. The parsing of $Y_{1} \ldots Y_{t}$ into blocks is already defined (by the expansion of $Y$ ), so we only need to copy it while adjusting the left border in order to include $X$. We distinguish two cases. If $Y_{1}$ and $Y_{2}$ are grouped into a single block, then we replace this block with the new block $X Y_{1} Y_{2}$ and collapse it in a new nonterminal at level $k_{X}+1$. If, on the other hand, $Y_{1}, Y_{2}$, and $Y_{3}$ are grouped into a single block then we replace it with the two blocks $X Y_{1}$ and $Y_{2} Y_{3}$ and collapse them in two new nonterminals at level $k_{X}+1$. We repeat the same procedure at levels $k_{X}+1, k_{X}+2, \ldots, k_{Y}$, until everything is collapsed in a single nonterminal. At each level we introduce one or two new nonterminals, therefore overall we introduce at most $2\left(k_{Y}-k_{X}\right)+1 \in O(\log (n / \gamma))$ new nonterminals. The case $k_{X}>k_{Y}$ is solved analogously except there is no upper bound of $n / \gamma$ on the length of the expansion of $X$ and hence in the worst case the procedure introduces $2\left(k_{X}-k_{Y}\right)+1 \in O(\log n)$ new nonterminals. Overall, however, this procedure generates the SLP for $T$ of size $O(\gamma \log (n / \gamma) \log (n))$.

To address the problem above we introduce $\gamma$ special blocks of size $2 n / \gamma$ starting at text positions that are multiples of $n / \gamma$, and we change the invariant ensuring that any contiguous sequence of already processed phrases is an expansion of some nonterminal, and instead require that at any point during the computation the invariant holds within all special blocks; more precisely, if for any special block we consider the smallest contiguous sequence $P_{1} \cdots P_{t}$ of phrases that overlaps both its endpoints (the endpoints of the block, that is), then the old invariant applied to any contiguous subsequence of $P_{1} \cdots P_{t}$ of already processed phrases has to hold. This is enough to guarantee that during the algorithm the source of every phrase is always a substring of an expansion of some nonterminal, and whenever we merge two nonterminals $X Y$ they always both each expand to a substring of length $O(n / \gamma)$ which guarantees that the merging introduces $O(\log (n / \gamma))$ new nonterminals. Furthermore, it is easy to see that once a phrase has been processed, in order to maintain the new invariant, we now need to perform at most 6 mergings of nonterminals (as opposed to at most 2 from before the modification), since each phrase can overlap at most three special blocks. Finally, at the end of the construction we need to make sure the whole string $T$ is an expansion of some nonterminal. To achieve this we do $\log (\gamma \log (n / \gamma))$ rounds of a pairwise merging of nonterminals corresponding to adjacent phrases (in the first round) or groups of phrases (in latter rounds). This adds $O(\gamma \log (n / \gamma))$ nonterminals. The level of the nonterminal expanding to $T$ (i.e., the height of the resulting SLP) is $O(\log (\gamma \log (n / \gamma))+\log (n / \gamma))=O(\log n)$.

Using the above theorems, we can derive the approximation rates of some compressors for repetitive strings with respect to the smallest string attractor.

Corollary 3.15. The following bounds hold between the size $g^{*}$ of the smallest SLP, the size $g_{r l}^{*}$ of the smallest run-length SLP, the size $z$ of the Lempel-Ziv parse, the size $b^{*}$ of the smallest macro scheme, the size $c^{*}$ of the smallest collage system, and the size $\gamma^{*}$ of the smallest string attractor:

(1) $b^{*}, c^{*} \in O\left(\gamma^{*} \log \left(n / \gamma^{*}\right)\right)$,

(2) $g^{*}, g_{r l}^{*}, z \in O\left(\gamma^{*} \log ^{2}\left(n / \gamma^{*}\right)\right)$.

Proof. For the first bounds, build the bidirectional parse of Theorem 3.12 and the collage system of Theorem 3.13 using a string attractor of minimum size $\gamma^{*}$. For the second bound, use the same attractor to build the SLP of Theorem 3.14 and exploit the well-known relation $z \leq g^{*}$ [39].

Our reductions and the above corollary imply our first approximation algorithms for the smallest string attractor. Note that only one of our approximations is computable in polynomial time (unless $\mathrm{P}=\mathrm{NP}$ ): the attractor induced by the LZ77 parsing. In the next section we show how to obtain asymptotically better approximations in polynomial time.

All our reductions combined imply the following relations between repetitiveness measures:

Corollary 3.16. The following bounds hold between the size $g^{*}$ of the smallest SLP, the size $z$ of the Lempel-Ziv parse, the size $c^{*}$ of the smallest collage system, the size $b^{*}$ of the smallest macro scheme, and the number $r$ of equal-letter runs in the BWT:

(1) $z, g^{*} \in O\left(b^{*} \log ^{2} \frac{n}{b^{*}}\right) \cap O\left(r \log ^{2} \frac{n}{r}\right) \cap O\left(c^{*} \log ^{2} \frac{n}{c^{*}}\right)$,

(2) $c^{*} \in O\left(b^{*} \log \frac{n}{b^{*}}\right) \cap O\left(r \log \frac{n}{r}\right)$,

(3) $b^{*} \in O\left(c^{*} \log \frac{n}{c^{*}}\right)$.

Proof. For bounds 1, build the SLP of Theorem 3.14 on string attractors of size $b^{*}, r$, and $c^{*}$ induced by the smallest macro scheme (Theorem 3.6), RLBWT (Theorem 3.9), and smallest collage system (Theorem 3.5). The results follow from the definition of the smallest SLP and the bound $z \leq g^{*}$ [39]. Similarly, bounds 2 and 3 are obtained with the reductions of Theorems 3.9, 3.12, and 3.13.

Some of these (or even tighter) bounds have been very recently obtained by Gagie et al. in [20] and in the extended version [19] of [21] using different techniques based on locally-consistent parsing. Our reductions, one the other hand, are slightly simpler and naturally include a broader class of dictionary compressors, e.g., all relations concerning $c^{*}$ have not been previously known.

\section{COMPUTATIONAL COMPLEXITY}

By Aтtractor $=\{\langle T, p\rangle:$ String $T$ has an attractor of size $\leq p\}$ we denote the language corresponding to the decision version of the smallest attractor problem. To prove the NP-completeness of Aтtractor we first generalize the notion of string attractor. 
Definition 4.1. We say that a set $\Gamma \subseteq[1 . . n]$ is a $k$-attractor of a string $T \in \Sigma^{n}$ if every substring $T[i . . j]$ such that $i \leq j<i+k$ has an occurrence $T\left[i^{\prime} . . j^{\prime}\right]=T[i . . j]$ with $j^{\prime \prime} \in\left[i^{\prime} . . j^{\prime}\right]$ for some $j^{\prime \prime} \in \Gamma{ }^{3}$

By Minimum- $k$-Atтractor we denote the optimization problem of finding the smallest $k$-attractor of a given input string. By

$k$-ATtractor $=\{\langle T, p\rangle: T$ has a $k$-attractor of size $\leq p\}$

we denote the corresponding decision problem. Observe that ATTRACTOR is a special case of $k$-ATTRACTOR where $k=n$. The NPcompleteness of $k$-ATtRACTOR for any $k \geq 3$ (this includes any constant $k \geq 3$ as well as any non-constant $k$ ) is obtained by a reduction from the $k$-SETCOVER problem that is NP-complete [17] for any constant $k \geq 3$ : given integer $p$ and a collection $C=\left\{C_{1}, C_{2}, \ldots, C_{m}\right\}$ of $m$ subsets of a universe set $\mathcal{U}=\{1,2, \ldots, u\}$ such that $\bigcup_{i=1}^{m} C_{i}=$ $\mathcal{U}$, and for any $i \in\{1, \ldots, m\},\left|C_{i}\right| \leq k$, return "yes" iff there exists a subcollection $C^{\prime} \subseteq C$ such that $\cup C^{\prime}=\mathcal{U}$ and $\left|C^{\prime}\right| \leq p$.

We obtain our reduction as follows. For any constant $k \geq 3$, given an instance $\langle\mathcal{U}, C\rangle$ of $k$-SETCover we build a string $T_{C}$ of length $O\left(u k^{2}+t k+t^{\prime}\right)$ where $t=\sum_{i=1}^{m} n_{i}$ and $t^{\prime}=\sum_{i=1}^{m} n_{i}^{2}$ with the following property: $\langle\mathcal{U}, C\rangle$ has a cover of size $\leq p$ if and only if $T_{C}$ has a $k$-attractor of size $\leq 4 u(k-1)+p+6 t-3 m$. This establishes the NP-completeness of $k$-ATTRACTOR for any constant $k \geq 3$. We then show that for $T_{C}$ the size of the smallest $k$-attractor is equal to that of the smallest $k^{\prime}$-attractor for every $k \leq k^{\prime} \leq\left|T_{C}\right|$, which allows us to prove the NP-completeness for non-constant $k$.

\section{Theorem 4.2. For $k \geq 3, k$-AtTRACTOR is NP-complete.}

Proof. Assume first that $k \geq 3$ is constant. We show a polynomial time reduction from $k$-SetCover to $k$-Atrractor. ${ }^{4}$ Denote the sizes of individual sets in the collection $C$ by $n_{i}=\left|C_{i}\right|>0$ and let $C_{i}=\left\{c_{i, 1}, c_{i, 2}, \ldots, c_{i, n_{i}}\right\}$. Recall that $u=|\mathcal{U}|$ and $m=|C|$.

Let

$$
\begin{aligned}
\Sigma= & \bigcup_{i=1}^{u} \bigcup_{j=1}^{k}\left\{x_{i}^{(j)}\right\} \cup \bigcup_{i=1}^{m} \bigcup_{j=1}^{n_{i}+1}\left\{\$_{i, j}\right\} \cup \bigcup_{i=1}^{u} \bigcup_{j=2}^{k}\left\{\$_{i, j}^{\prime}, \$_{i, j}^{\prime \prime}\right\} \cup \\
& \bigcup_{i=1}^{m} \bigcup_{j=2}^{n_{i}}\left\{\$_{i, j}^{\prime \prime \prime}, \$_{i, j}^{(4)}\right\} \cup\{\#\}
\end{aligned}
$$

be our alphabet. Note that in the construction below, $x_{i}^{(j)}$ or $\$_{i, j}^{(4)}$ denotes a single symbol, while $\#^{k-1}$ denotes a concatenation of $k-1$ occurrences of symbol \#. We will now build a string $T_{C}$ over the alphabet $\Sigma$.

Let

$$
T_{C}=\prod_{i=1}^{u} P_{i} \cdot \prod_{i=1}^{m} R_{i} S_{i},
$$

where $\cdot / \Pi$ denotes the concatenation of strings and $P_{i}, R_{i}$, and $S_{i}$ are defined below.

Intuitively, we associate each $t \in \mathcal{U}$ with the substring $x_{t}^{(1)} \cdots x_{t}^{(k)}$ and each collection $C_{i}$ with $S_{i}$. Each $S_{i}$ will contain all $n_{i}$ strings corresponding to elements of $C_{i}$ as substrings. The aim of $S_{i}$ is to simulate - via how many positions are used within $S_{i}$ in the solution to the $k$-Аттractor on $T_{C}$ - the choice between not including

\footnotetext{
${ }^{3}$ We permit non-constant $k=f(n)$ where $n=|T|$ as long as $\lim _{n \rightarrow \infty} f(n)=\infty$ and $f(n)$ is non-decreasing.

${ }^{4}$ The proof only requires that $k \geq 3$ but we point out that the reduction is valid also for $k=2$.
}

$C_{i}$ in the solution to $k$-SETCover on $C$ (in which case $S_{i}$ is covered using a minimum possible number of positions that necessarily leaves uncovered all substrings corresponding to elements of $C_{i}$ ) or including $C_{i}$ (in which case, by using only one additional position in the cover of $S_{i}$, the solution covers all substrings unique to $S_{i}$ and simultaneously all $n_{i}$ substrings of $S_{i}$ corresponding to elements of $C_{i}$ ). Gadgets $R_{i}$ and $P_{i}$ are used to cover "for free" certain substrings occurring in $S_{i}$ so that any algorithm solving $k$-ATTRACTOR for $T_{C}$ will not have to optimize for their coverage within $S_{i}$. This will be achieved as follows: each gadget $P_{i}$ (similar for $R_{i}$ ) will have $x_{P_{i}}$ non-overlapping substrings (for some $x_{P_{i}}$ ) that appear only in $P_{i}$ and nowhere else in $T_{C}$. This will imply that any $k$-attractor for $T_{C}$ has to include at least $x_{P_{i}}$ positions within $P_{i}$. On the other hand, we will show that there exists an optimal choice of $x_{P_{i}}$ positions within $P_{i}$ that covers all those unique substrings, plus the substrings of $P_{i}$ occurring also $S_{i}$ that we want to cover "for free" within $P_{i}$.

For $i \in\{1,2, \ldots, m\}$, let (brackets added for clarity)

$$
S_{i}=\left(\prod_{j=1}^{n_{i}} \#^{k-1} \$_{i, 1} \cdots \$_{i, j} x_{c_{i, j}}^{(1)} \cdots x_{c_{i, j}}^{(k)} \$_{i, j}\right) \#^{k-1} \$_{i, 1} \cdots \$_{i, n_{i}+1} \text {. }
$$

An example of $S_{i}$ for $k=6$ and $n_{i}=4$ is reported below. The meaning of overlined and underlined characters is explained next.

$$
\begin{aligned}
& S_{i}=\# \# \# \# \Phi_{i, 1} \overline{x_{c_{i, 1}}^{(1)}} x_{c_{i, 1}}^{(2)} x_{c_{i, 1}}^{(3)} x_{c_{i, 1}}^{(4)} x_{c_{i, 1}}^{(5)} x_{c_{i, 1}}^{(6)} \overline{\$_{i, 1}} \\
& \text { \#\#\#\#\$ }{ }_{i, 1} \underline{\$_{i, 2}} \bar{x}_{c_{i, 2}^{(1)}}^{(2)} x_{c_{i, 2}}^{(2)} x_{c_{i, 2}}^{(3)} x_{c_{i, 2}}^{(4)} x_{c_{i, 2}}^{(5)} x_{c_{i, 2}}^{(6)} \underline{\$_{i, 2}} \\
& \text { \#\#\#\#\$ }{ }_{i, 1} \$_{i, 2} \$_{i, 3} \overline{x_{c_{i, 3}}^{(1)}} x_{c_{i, 3}}^{(2)} x_{c_{i, 3}}^{(3)} x_{c_{i, 3}}^{(4)} x_{c_{i, 3}}^{(5)} x_{c_{i, 3}}^{(6)} \overline{\$_{i, 3}} \\
& \text { \#\#\#\#\$ } \$_{i, 1} \$_{i, 2} \$_{i, 3} \$_{i, 4} \overline{x_{c_{i, 4}}^{(1)}} x_{c_{i, 4}}^{(2)} x_{c_{i, 4}}^{(3)} x_{c_{i, 4}}^{(4)} x_{c_{i, 4}}^{(5)} x_{c_{i, 4}}^{(6)} \overline{\$_{i, 4}} \\
& \# \# \# \overline{\$_{i, 1}} \$_{i, 2} \$_{i, 3} \$_{i, 4} \overline{\$_{i, 5}}
\end{aligned}
$$

Any $k$-attractor of $T_{C}$ contains at least $2 n_{i}+1$ positions within $S_{i}$ because: (i) $S_{i}$ contains $2 n_{i}$ non-overlapping substrings of length $k$, each of which necessarily $y^{5}$ occurs in $S_{i}$ only once and nowhere else ${ }^{6}$ in $T_{C}$ :

$$
\bigcup_{j=1}^{n_{i}}\left\{\$_{i, j} \#^{k-1}\right\} \cup \bigcup_{j=1}^{n_{i}}\left\{\$_{i, j} x_{c_{i, j}}^{(1)} \cdots x_{c_{i, j}}^{(k-1)}\right\}
$$

and (ii) $S_{i}$ contains symbol $\$_{i, n_{i}+1}$, which occurs only once in $S_{i}$ and nowhere else in $T_{C}$, and does not overlap any of the $2 n_{i}$ substrings mentioned before. With this in mind we now observe that $S_{i}$ has the following two properties:

(1) There exists a "minimum" set $\Gamma_{S, i}$ of $2 n_{i}+1$ positions within the occurrence of $S_{i}$ in $T_{C}$ that covers all substrings of $S_{i}$ of length $\leq k$ that necessarily occur only in $S_{i}$ and nowhere else in $T_{C}$. The set $\Gamma_{S, i}$ includes: the leftmost occurrence of $\$_{i, j}$ for $j \in\left\{1, \ldots, n_{i}+1\right\}$ and the second occurrence from the left of $\$_{i, j}$ for $j \in\left\{1, \ldots, n_{i}\right\}\left(\Gamma_{S, i}\right.$ is shown in the above example using underlined positions). Furthermore, $\Gamma_{S, i}$ is the only such set. This is because in any such set there needs to be at

\footnotetext{
That is, independent of what is $C_{i}$. Importantly, although any of the substrings in $\bigcup_{j=1}^{n_{i}}\left\{x_{c_{i, j}}^{(1)} \cdots x_{c_{i, j}}^{(k)}\right\}$ could have the only occurrence in $S_{i}$, they are not necessarily unique to $S_{i}$. This situation is analogous to $k$-SETCover when some $t \in \mathcal{U}$ is covered by only one set in $C$, and thus that set has to be included in the solution.

${ }^{6}$ This can be verified by consulting the definitions of families $\left\{R_{t}\right\}_{t=1}^{m}$ and $\left\{P_{t}\right\}_{t=1}^{u}$ that follow.
} 
least one position inside each of the $2 n_{i}+1$ non-overlapping substrings of $S_{i}$ mentioned above. In the first $n_{i}$ of those substrings, $\bigcup_{j=1}^{n_{i}}\left\{\$_{i, j} \#^{k-1}\right\}$, the first position intersects $k$ distinct substrings of length $k$ that necessarily occur only once in $S_{i}$ and nowhere else in $T_{C}$, and hence in those substrings the position in the attractor is fixed. Next, the position in any such set is also trivially fixed for the only occurrence of $\$_{i, n_{i}+1}$ in $T_{C}$. Let us then finally look at each of the remaining $n_{i}$ substrings, $\bigcup_{j=1}^{n_{i}}\left\{\$_{i, j} x_{c_{i, j}}^{(1)} \cdots x_{c_{i, j}}^{(k-1)}\right\}$, starting from the rightmost $\left(j=n_{i}\right)$. In the substring $\$_{i, n_{i}} x_{c_{i, n_{i}}}^{(1)} \cdots x_{c_{i, n_{i}}}^{(k-1)}$ the first position intersects only $k-1$ substrings of $S_{i}$ of length $k$ that necessarily occur only once in $S_{i}$ and nowhere else in $T_{C}$. However, all other occurrences (for $j=n_{i}$ there is only one; in general there is $n_{i}-j+1$ occurrences) in $T_{C}$ of the remaining non-unique substring intersecting the first position, $\#^{k-n_{i}} \$_{i, 1} \cdots \$_{i, n_{i}}$, are in $S_{i}$ (and nowhere else in $T_{C}$ ), to the right of the discussed occurrence of $\$_{i, n_{i}} x_{c_{i, n_{i}}}^{(1)} \cdots x_{c_{i, n_{i}}}^{(k-1)}$, and are not covered. Thus, the attractor needs to include the first position in this substring. Repeating this argument for $j=n_{i}-1, \ldots, 1$ yields the claim. Now we observe that the only substrings of $S_{i}$ of length $\leq k$ not covered by $\Gamma_{S, i}$ are strings $\left\{\#^{k-1}\right\} \cup \bigcup_{j=1}^{n_{i}}\left\{x_{c_{i, j}}^{(1)} \cdots x_{c_{i, j}}^{(k)}\right\}$ and all their proper substrings. We have thus demonstrated that if in any $k$-attractor of $T_{C}, S_{i}$ is covered using the minimum number of $2 n_{i}+1$ positions, these positions must be precisely $\Gamma_{S, i}$ and hence, in particular, any of the strings in the set $\bigcup_{j=1}^{n_{i}}\left\{x_{c_{i, j}}^{(1)} \cdots x_{c_{i, j}}^{(k)}\right\}$ is then not covered within $S_{i}$.

(2) There exists a "nearly-universal" set $\Gamma_{S, i}^{\prime}$ of $2 n_{i}+2$ positions within the occurrence of $S_{i}$ in $T_{C}$ that covers: (i) all substrings of $S_{i}$ of length $\leq k$ that necessarily occur only in $S_{i}$ and nowhere else in $T_{C}$, and (ii) $\bigcup_{j=1}^{n_{i}}\left\{x_{c_{i, j}}^{(1)} \cdots x_{c_{i, j}}^{(k)}\right\}$. The set $\Gamma_{S, i}^{\prime}$ includes: the only occurrence of $x_{c_{i, j}}^{(1)}$ for $j \in\left\{1, \ldots, n_{i}\right\}$, the second occurrence of $\$_{i, j}$ for $j \in\left\{1, \ldots, n_{i}\right\}$, the only occurrence of $\$_{i, n_{i}+1}$, and the last occurrence of $\$_{i, 1}\left(\Gamma_{S, i}^{\prime}\right.$ is shown in the above example using overlined positions). The only substrings of $S_{i}$ of length $\leq k$ not covered by $\Gamma_{S, i}^{\prime}$ are strings $\left\{\#^{k-1}\right\} \cup \bigcup_{j=1}^{n_{i}}\left\{x_{c_{i, j}}^{(2)} \cdots x_{c_{i, j}}^{(k)}\right\}$ and all their proper substrings, and all substrings of length $>1$ of the string $\$_{i, 2} \cdots \$_{i, n_{i}}$. For these strings we introduce separate gadget strings described next.

To finish the construction, we will ensure that for any $i \in$ $\{1, \ldots, m\}$, certain substrings of $S_{i}$ are covered "for free" elsewhere in $T_{C}$. To this end we introduce families $\left\{P_{i}\right\}_{i=1}^{u}$ and $\left\{R_{i}\right\}_{i=1}^{m}$. Specifically, all strings (and all their proper substrings) in the set $\left\{\#^{k-1}\right\} \cup$ $\bigcup_{i=1}^{u}\left\{x_{i}^{(2)} \cdots x_{i}^{(k)}\right\}$ will be covered for free in $\left\{P_{i}\right\}_{i=1}^{u}$. Analogously, all strings (and all their proper substrings) in $\bigcup_{i=1}^{m}\left\{\$_{i, 2} \cdots \$_{i, n_{i}}\right\}$ will be covered for free in $\left\{R_{i}\right\}_{i=1}^{m}$. Assuming these substrings are covered: (i) if we use $\Gamma_{S, i}$ to cover unique substrings of $S_{i}$, the only substrings of $S_{i}$ of length $\leq k$ not covered by $\Gamma_{S, i}$ will be $\bigcup_{j=1}^{n_{i}}\left\{x_{c_{i, j}}^{(1)} \cdots x_{c_{i, j}}^{(k)}\right\}$, and (ii) if we use $\Gamma_{S, i}^{\prime}$, all substrings of $S_{i}$ of length $\leq k$ will be covered.
We now show the existence of the families $\left\{P_{i}\right\}_{i=1}^{u}$ and $\left\{R_{i}\right\}_{i=1}^{m}$. For $i \in\{1,2, \ldots, u\}$, let

$$
P_{i}=\prod_{j=2}^{k} \#^{k-1} \$_{i, j}^{\prime} x_{i}^{(2)} \cdots x_{i}^{(j)} \$_{i, j}^{\prime \prime} \#^{k-1} \$_{i, j}^{\prime} x_{i}^{(2)} \cdots x_{i}^{(j)} \$_{i, j}^{\prime} \$_{i, j}^{\prime} \$_{i, j}^{\prime \prime}
$$

A prefix of $P_{i}$ for $k=6$ is

$$
\begin{aligned}
& P_{i}=\# \# \# \# \$_{i, 2}^{\prime} x_{i}^{(2)} \$_{i, 2}^{\prime \prime} \# \# \# \# \$_{i, 2}^{\prime} x_{i}^{(2)} \underline{\$_{i, 2}^{\prime}} \$_{i, 2}^{\prime} \underline{\$_{i, 2}^{\prime \prime}} \\
& \text { \#\#\#\#\# } \$_{i, 3}^{\prime} x_{i}^{(2)} x_{i}^{(3)} \$_{i, 3}^{\prime \prime} \# \# \# \# \$_{i, 3}^{\prime} x_{i}^{(2)} x_{i}^{(3)} \underline{\$_{i, 3}^{\prime}} \$_{i, 3}^{\prime} \$_{i, 3}^{\prime \prime} \\
& \text { \#\#\#\#\# } \$_{i, 4}^{\prime} x_{i}^{(2)} x_{i}^{(3)} x_{i}^{(4)} \$_{i, 4}^{\prime \prime} \# \# \# \# \$_{i, 4}^{\prime} x_{i}^{(2)} x_{i}^{(3)} x_{i}^{(4)} \$_{i, 4}^{\prime} \$_{i, 4}^{\prime} \$_{i, 4}^{\prime \prime} .
\end{aligned}
$$

Any $k$-attractor of $T_{C}$ contains at least $4(k-1)$ positions within $P_{i}$ because there are $4(k-1)$ non-overlapping substrings of length two of $P_{i}$ that occur only in $P_{i}$ and nowhere else in $T_{C}{ }^{7}$ These substrings are, for $j \in\{2, \ldots, k\}: \# \$_{i, j}^{\prime}, x_{i}^{(j)} \$_{i, j}^{\prime \prime}, x_{i}^{(j)} \$_{i, j}^{\prime}, \$_{i, j}^{\prime} \$_{i, j}^{\prime \prime}$.

On the other hand, there exists a "universal" set $\Gamma_{P, i}$ of $4(k-1)$ positions within the occurrence $P_{i}$ in $T_{C}$ that covers all substrings of $P_{i}$ of length $\leq k .^{8}$ In particular, $\Gamma_{P, i}$ covers the strings $x_{i}^{(2)} \cdots x_{i}^{(k)}$ and $\#^{k-1}$, and all their proper substrings. The set $\Gamma_{P, i}$ includes: the position of the leftmost occurrence of $x_{i}^{(j)}$ for $j \in\{2, \ldots, k\}$, the position preceding the second occurrence of $\$_{i, 2}^{\prime}$ from the left, the third occurrence of $\$_{i, 2}^{\prime}$ from the left, the second and third occurrences of $\$_{i, j}^{\prime}$ from the left for $j \in\{3, \ldots, k\}$, and the second occurrence of $\$_{i, j}^{\prime \prime}$ from the left for $j \in\{2, \ldots, k\}$. The positions in $\Gamma_{P, i}$ are underlined in the above example.

For $i \in\{1,2, \ldots, m\}$, let

$$
R_{i}=\prod_{j=2}^{n_{i}} \#^{k-1} \$_{i, j}^{\prime \prime \prime} \$_{i, 2} \cdots \$_{i, j} \$_{i, j}^{(4)} \#^{k-1} \$_{i, j}^{\prime \prime \prime} \$_{i, 2} \cdots \$_{i, j} \$_{i, j}^{\prime \prime \prime} \$_{i, j}^{\prime \prime \prime} \$_{i, j}^{(4)} \text {. }
$$

An example of $R_{i}$ for $k=6$ and $n_{i}=4$ is

$$
\begin{aligned}
& R_{i}=\# \# \# \# \$_{i, 2}^{\prime \prime \prime} \$_{i, 2} \$_{i, 2}^{(4)} \# \# \# \# \$_{i, 2}^{\prime \prime \prime} \$_{i, 2} \$_{i, 2}^{\prime \prime \prime} \$_{i, 2}^{\prime \prime \prime} \$_{i, 2}^{(4)} \\
& \text { \#\#\#\#\# } \$_{i, 3}^{\prime \prime \prime} \$_{i, 2} \$_{i, 3} \$_{i, 3}^{(4)} \# \# \# \# \$_{i, 3}^{\prime \prime \prime} \$_{i, 2} \$_{i, 3} \$_{i, 3}^{\prime \prime \prime} \$_{i, 3}^{\prime \prime \prime} \$_{i, 3}^{(4)} \\
& \# \# \# \# \$_{i, 4}^{\prime \prime \prime} \$_{i, 2} \$_{i, 3} \$_{i, 4} \$_{i, 4}^{(4)} \# \# \# \# \$_{i, 4}^{\prime \prime \prime} \$_{i, 2} \$_{i, 3} \$_{i, 4} \$_{i, 4}^{\prime \prime \prime} \$_{i, 4}^{\prime \prime \prime} \$_{i, 4}^{(4)} .
\end{aligned}
$$

Note, that if $n_{i}=1$ then $R_{i}$ is the empty string. Suppose that $R_{i}$ is non-empty, i.e., $n_{i} \geq 2$. The construction of $R_{i}$ is analogous to $P_{i}$. Any $k$-attractor of $T_{C}$ contains at least $4\left(n_{i}-1\right)$ positions within $R_{i}$. On the other hand, there exists a "universal" set $\Gamma_{R, i}$ of $4\left(n_{i}-1\right)$ positions of $R_{i}$ that covers all substrings of $R_{i}$ of length $\leq k$. In particular, $\Gamma_{R, i}$ covers the string $\$_{i, 2} \cdots \$_{i, n_{i}}$ and all its proper substrings. The set $\Gamma_{R, i}$ includes: the position of the leftmost occurrence of $\$_{i, j}$ for $j \in\left\{2, \ldots, n_{i}\right\}$, the position preceding the second occurrence of $\$_{i, 2}^{\prime \prime \prime}$ from the left, the third occurrence of $\$_{i, 2}^{\prime \prime \prime}$ from the left, the second and third occurrence of $\$_{i, j}^{\prime \prime \prime}$ for $j \in$ $\left\{3, \ldots, n_{i}\right\}$, and the second occurrence of $\$_{i, j}^{(4)}$ from the left for $j \in\left\{2, \ldots, n_{i}\right\}$. The positions in $\Gamma_{R, i}$ are underlined in the example.

\footnotetext{
${ }^{7}$ This for example enforces $k \geq 2$ in our proof.

${ }^{8}$ Note a small subtlety here. Because each of the gadgets $\left\{P_{i}\right\}_{i=1}^{u},\left\{S_{i}\right\}_{i=1}^{m},\left\{R_{i}\right\}_{i=1}^{m}$ begins with $\#^{k-1}$ and each $P_{i}$ is followed by some other gadget in $T_{C}$, the following set of substrings of $P_{i}:\left\{\$_{i, k}^{\prime \prime} \#^{t}\right\}_{t=1}^{k-1}$ will indeed be covered by $\Gamma_{P, i}$ but for $k>2$ the covered occurrences are not substrings of $P_{i}$. An analogous property holds for $\left\{R_{i}\right\}_{i=1}^{m}$.
} 
With the above properties, we are now ready to prove the following claim: an instance $\langle\mathcal{U}, C\rangle$ of $k$-SetCover has a solution of size $\leq p$ if and only if $T_{C}$ has a $k$-attractor of size $\leq 4 u(k-1)+p+6 t-3 m$, where $t=\sum_{i=1}^{m} n_{i}$.

“ $(\Rightarrow)$ " Let $C^{\prime} \subseteq C$ be a cover of $\mathcal{U}$ of size $p^{\prime} \leq p$ and let

$\Gamma_{C^{\prime}}=\bigcup\left\{\Gamma_{S, i}^{\prime} \mid C_{i} \in C^{\prime}\right\} \cup \bigcup\left\{\Gamma_{S, i} \mid C_{i} \notin C^{\prime}\right\} \cup \bigcup_{i=1}^{u} \Gamma_{P, i} \cup \bigcup_{i=1}^{m} \Gamma_{R, i}$.

$\Gamma_{C^{\prime}}$ contains universal attractors $\Gamma_{P}$. and $\Gamma_{R}$, introduced above for $\left\{P_{i}\right\}_{i=1}^{u}$ and $\left\{R_{i}\right\}_{i=1}^{m}$, and nearly-universal attractors $\Gamma_{S}^{\prime}$, for elements of $\left\{S_{i}\right\}_{i=1}^{m}$ corresponding to elements of $C^{\prime}$. All other strings in $\left\{S_{i}\right\}_{i=1}^{m}$ are covered using minimum attractors $\Gamma_{S}, .$. It is easy to check that $\left|\Gamma_{C^{\prime}}\right|=4 u(k-1)+p^{\prime}+6 t-3 m$. From the above discussion $\Gamma_{C^{\prime}}$ covers all substrings of $T_{C}$ of length $\leq k$ inside $\left\{P_{i}\right\}_{i=1}^{u},\left\{R_{i}\right\}_{i=1}^{m}$, and $\left\{S_{i}\right\}_{i=1}^{m}$. In particular, $\left\{x_{i}^{(1)} \cdots x_{i}^{(k)}\right\}_{i=1}^{u}$ are covered because $C^{\prime}$ is a cover of $\mathcal{U}$. All other substrings of $T_{C}$ of length $\leq k$ span at least two gadget strings and thus are also covered since all sets forming $\Gamma_{C^{\prime}}$ include the last position of the gadget string.

" $(\Leftarrow)$ " Let $\Gamma$ be a $k$-attractor of $T_{C}$ of size $\leq 4 u(k-1)+p+6 t-3 m$. We will show that $\mathcal{U}$ must have a cover of size $\leq p$ using elements from $C$. Let $\mathcal{I}$ be the set of indices $i \in\{1, \ldots, m\}$ for which $\Gamma$ contains more than $2 n_{i}+1$ positions within the occurrence of $S_{i}$ in $T_{C}$. To bound the cardinality of $\mathcal{I}$ we first observe that by the above discussion, $\Gamma$ cannot have less than $\sum_{i=1}^{m} 4\left(n_{i}-1\right)+\sum_{i=1}^{u} 4(k-1)=$ $4 u(k-1)+4 t-4 m$ positions within all occurrences of $\left\{P_{i}\right\}_{i=1}^{u}$ and $\left\{R_{i}\right\}_{i=1}^{m}$ in $T_{C}$. Thus, there is only at most $2 t+m+p$ positions left to use within $\left\{S_{i}\right\}_{i=1}^{m}$. Furthermore, each of $S_{i}, i \in\{1, \ldots, m\}$ requires $2 n_{i}+1$ positions, and hence there cannot be more than $p$ indices where $\Gamma$ uses more positions than necessary. Thus, $|\mathcal{I}| \leq p$. Let $C_{\Gamma}^{\prime}=\left\{C_{i} \in C \mid i \in \mathcal{I}\right\}$. We now show that $C_{\Gamma}^{\prime}$ is a cover of $\mathcal{U}$. Take any $t \in \mathcal{U}$. Since $\Gamma$ is a $k$-attractor of $T_{C}$, the string $x_{t}^{(1)} \cdots x_{t}^{(k)}$ is covered in some $S_{i_{t}}$ such that $t \in C_{i_{t}}$. By the above discussion for this to be possible $\Gamma$ must use more than $2 n_{i_{t}}+1$ positions within $S_{i_{t}}$. Thus, $i_{t} \in \mathcal{I}$ and hence $C_{i_{t}} \in C_{\Gamma}^{\prime}$.

The above reduction proves the NP-completeness of $k$-ATTRACTOR for any constant $k \geq 3$. We now show a property of $T_{C}$ that will allow us to obtain the NP-completeness for non-constant $k$. Denote the size of the smallest $k$-attractor of string $X$ by $\gamma_{k}^{*}(X)$. By definition a $k^{\prime}$-attractor of string $X$ is also a $k$-attractor of $X$ for any $k<k^{\prime}$ and thus for any $k \in\{1, \ldots,|X|-1\}, \gamma_{k}^{*}(X) \leq \gamma_{k+1}^{*}(X)$. The inequality in general can be strict, e.g., for $X=$ acacaacc, $\gamma_{2}^{*}(X)<\gamma_{3}^{*}(X)$. We now show that for $T_{C}$ it holds $\gamma_{k}^{*}\left(T_{C}\right)=\gamma_{k^{\prime}}^{*}\left(T_{C}\right)$ for any $k<k^{\prime} \leq\left|T_{C}\right|$. Assume that $p$ is the size of the smallest $k$-set-cover of $\mathcal{U}$ and let $C^{\prime} \subseteq C$ be the optimal cover. Then, $\Gamma_{C^{\prime}}$ (defined as above) is the smallest $k$-attractor of $T_{C}$ and, crucially, admits a particular structure, namely, it is a union of universal, nearly-universal and minimum attractors introduced above. We will now show that $\Gamma_{C^{\prime}}$ is a $k^{\prime}$-attractor of $T_{C}$. Since each of the sets forming $\Gamma_{C^{\prime}}$ covers the last position of the corresponding gadget string, we can focus on substrings of length $>k$ entirely contained inside gadget strings. To show the claim for $\left\{S_{i}\right\}_{i=1}^{m}$ it suffices to verify that all substrings of $\#^{k-1} \$_{i, 1} \cdots \$_{i, n_{i}}$ of length $>k$ are covered in both $\Gamma_{S, i}$ and $\Gamma_{S, i}^{\prime}$. Analogously, for $\left\{P_{i}\right\}_{i=1}^{u}$ and $\left\{R_{i}\right\}_{i=1}^{m}$ it suffices to verify the claim for the families $\left\{\#^{k-1} \$_{i, j}^{\prime} x_{i}^{(2)} \cdots x_{i}^{(j-1)}\right\}_{j=3}^{k}$ and $\left\{\#^{k-1} \$_{i, j}^{\prime \prime \prime} \$_{i, 2} \cdots \$_{i, j-1}\right\}_{j=3}^{n_{i}}$. Thus, $\Gamma_{C^{\prime}}$ is a $k^{\prime}$-attractor of $T_{C}$.
To show the NP-completeness of $k$-ATTRACTOR for non-constant $k$ (in particular for $k=n$ ) consider any non-decreasing function $k=f(n)$ such that $\lim _{n \rightarrow \infty} f(n)=\infty$. Let $n_{0}=\min \{n \geq 1 \mid$ $f(n) \geq 3\}$. Suppose that we have a polynomial-time algorithm for $f(n)$-AtTractor. Consider an instance $\langle\mathcal{U}, C\rangle, C=\left\{C_{i}\right\}_{i=1}^{m}$ of 3-SetCover. To decide if $\langle\mathcal{U}, C\rangle$ has a cover of size $\leq p$, we first build the string $T_{C}$. If $\left|T_{C}\right|<n_{0}$, we run a brute-force algorithm to find the answer in $O\left(2^{m}\right.$ poly $\left.(t)\right)=O\left(2^{n_{0}}\right.$ poly $\left.\left(n_{0}\right)\right)=O(1)$ time, where $t=\sum_{i=1}^{m}\left|C_{i}\right|$. Otherwise, the answer is given by checking the inequality $\gamma_{3}^{*}\left(T_{C}\right)=\gamma_{f\left(\left|T_{C}\right|\right)}^{*}\left(T_{C}\right) \leq 8 u+p+6 t-3 m$ (where $u=|\mathcal{U}|)$ in polynomial time.

We further demonstrate that Minimum- $k$-ATtRACTOR can be efficiently approximated up to a constant factor when $k \geq 3$ is constant, but unless $\mathrm{P}=\mathrm{NP}$, does not have a PTAS. This is achieved by a reduction from vertex cover on bounded-degree graphs, utilizing the smallest $k$-set cover as an intermediate problem. Using explicit constants derived by Berman and Karpinski [7] for the vertex cover, we also obtain explicit constants for our problem (and general $k$ ).

Theorem 4.3. For any constant $k \geq 3$, Minimum- $k$-ATtRACTOR is APX-complete.

Proof. Denote the size of the smallest $k$-attractor of $T$ by $\gamma_{k}^{*}(T)$ and let $\sigma_{k}(T)$ be the number of different substrings of $T$ of length $k$. We claim that $\gamma_{k}^{*}(T) \leq \sigma_{k}\left(T^{2}\right) \leq 2 k \gamma_{k}^{*}(T)$ (where $T^{2}$ is a concatenation of two copies of $T$ ). To show the first inequality, define $\Gamma$ as the set containing the beginning of the leftmost occurrence of every distinct substring of $T^{2}$ of length $k$. Such $\Gamma$ can be easily computed in polynomial time. We claim that $\Gamma$ is a $k$-attractor of $T$. Consider any substring of $T$ of length $k^{\prime} \leq k$ and let $T\left[i . . i+k^{\prime}-1\right]$ be its leftmost occurrence. Then, $T^{2}[i . . i+k-1]$ is the leftmost occurrence of $T^{2}[i . . i+k-1]$ in $T^{2}$, as otherwise we would have an earlier occurrence of $T\left[i . . i+k^{\prime}-1\right]$ in $T$. Thus, $i \in \Gamma$. On the other hand, each position in a $k$-attractor of $T^{2}$ covers at most $k$ distinct substrings of $T^{2}$ of length $k$. Thus $\gamma_{k}^{*}\left(T^{2}\right) \geq\left\lceil\sigma_{k}\left(T^{2}\right) / k\right\rceil$. Combining this with $\gamma_{k}^{*}\left(T^{2}\right) \leq \gamma_{k}^{*}(T)+1$ gives the second inequality. Thus, Minimum- $k$-AtTractor is in APX.

To show that MINIMUM- $k$-ATTRACTOR is APX-hard we generalize the hardness argument of Charikar et al. [12] from grammars to attractors. We show that to approximate MINIMUM- $k$-VerTexCover (minimum vertex cover for graphs with vertex-degree bounded by $k$ ) in polynomial time below a factor $1+\epsilon$, for any constant $\epsilon>0$, it suffices to approximate MINIMUM- $k$-ATTRACTOR in polynomial time below a factor $1+\delta$, where $\delta=\epsilon /\left(2 k^{3}+4 k^{2}-3 k+1\right)$. In other words, if Minimum- $k$-AtTractor has a PTAS then Minimum- $k$ VertexCover also has a PTAS. Since Minimum- $k$-VertexCover is APX-hard [37], this will yield the claim.

Let $G=(V, E)$ be an undirected graph with vertex-degree bounded by $k$. Assume that $|V| \leq|E|$ and that $G$ has no isolated vertices. ${ }^{9}$ Let $\mathcal{U}_{G}=E$ and $C_{G}=\left\{E_{v} \mid v \in V\right\}$, where $E_{v}=\{e \in E \mid$ $e$ is incident to $v$. Then, the size of the minimum $k$-set cover for $C_{G}$ is $p$ if and only if the minimum $k$-vertex cover of $G$ has size $p$. Consider the string $T_{G}:=T_{C_{G}}$ as in Theorem 4.2. The smallest $k$-attractor of $T_{G}$ has size $(8+4 k)|E|-3|V|+p$ (since the universe

${ }^{9}$ Minimum- $k$-VertexCover is still APX-hard under this assumption, since a PTAS for this case would give us a PTAS for the general case. 
size is $|E|$, the number of sets in $C_{G}$ is $|V|$, and their total cardinality is $2|E|$ ) if and only if the smallest vertex cover of $G$ has size $p$.

Assume it is NP-hard to approximate MINIMUM- $k$-VertexCover below the ratio $1+\epsilon$. Then it is also NP-hard to approximate the smallest $k$-attractor for $T_{G}$ below the ratio

$$
r=\frac{(8+4 k)|E|-3|V|+(1+\epsilon) p}{(8+4 k)|E|-3|V|+p}=1+\frac{\epsilon p}{(8+4 k)|E|-3|V|+p} .
$$

Since all vertices have degree at most $k, 2|E| \leq k|V|$. Furthermore, since each vertex can cover at most $k$ edges, the size of the minimum $k$-vertex cover, $p$, must be at least $\frac{1}{k}|E| \geq \frac{1}{k}|V|$. The expression above achieves its minimum when $|E|$ is large and $p$ is small. From the constraints $|E| \leq \frac{k}{2}|V|$ and $p \geq \frac{1}{k}|V|$, we thus get the lower bound

$$
r \geq 1+\frac{\epsilon \cdot \frac{1}{k}|V|}{(8+4 k) \cdot \frac{k}{2}|V|-3|V|+\frac{1}{k}|V|}=1+\frac{\epsilon}{2 k^{3}+4 k^{2}-3 k+1} .
$$

COROllary 4.4. For every constant $\epsilon>0$ and every (not necessarily constant) $k \geq 3$, it is NP-hard to approximate MINIMUM- $k$ ATTRACTOR within factor $11809 / 11808-\epsilon$.

Proof. By [7], Minimum-3-VertexCover is NP-hard to approximate below a factor $1+\epsilon_{3}=\frac{145}{144}$. By Theorem 4.3 it is equally hard to approximate Minimum-3-AtTractor below $1+\frac{\epsilon_{3}}{2 k^{3}+4 k^{2}-3 k+1}$, where $k=3$. The claim for larger (and non-constant) $k$ follows from the property $\gamma_{k}^{*}\left(T_{G}\right)=\gamma_{k^{\prime}}^{*}\left(T_{G}\right), k<k^{\prime} \leq\left|T_{G}\right|$ of the string $T_{G}$ used in the proof on Theorem 4.3.

Theorem 4.3 implies a $2 k$-approximation algorithm for MINIMUM$k$-Attractor, $k \geq 3$. By reducing the problem to Minimum- $k$ SETCOVER we can however obtain a better ratio.

TheOREM 4.5. For any $k \geq 3$, MINIMUM- $k$-ATTRACTOR can be approximated in polynomial time up to a factor of $\mathcal{H}(k(k+1) / 2)$, where $\mathcal{H}(p)=\sum_{i=1}^{p} \frac{1}{i}$ is the $p$-th harmonic number. In particular, MINIMUMATTRACTOR can be approximated to a factor $\mathcal{H}(n(n+1) / 2) \leq$ $2 \ln ((n+1) / \sqrt{2})+1$.

Proof. We first show that in polynomial time we can reduce Minimum- $k$-Attractor to an instance of Minimum- $k^{\prime}$-SetCover for $k^{\prime}=k(k+1) / 2$. Let $T$ be the input string of length $n$. Consider the set $\mathcal{U}$ of all distinct substrings of $T$ of length $\leq k$. The size of $\mathcal{U}$ is at most $k n$, i.e., polynomial in $n$. We create a collection $C$ of sets over $\mathcal{U}$ as follows. For any position $i \in[1, n]$ in $T$ take all distinct substrings of length $\leq k$ that have an occurrence containing position $i$ (there is at most $p$ such substrings of length $p$ and hence not more than $k(k+1) / 2$ in total $)$ and add a set containing those substrings to $C$. It is easy to see that MINIMUM- $k$-ATTRACTOR for $T$ has the same size as Minimum- $k^{\prime}$-SETCOVER for $C$. Since the latter can be approximated to a factor $\mathcal{H}\left(k^{\prime}\right)$ [24], the claim follows.

For constant $k \geq 3$, Duh and Fürer [17] describe an approximation algorithm based on semi-local optimization that achieves an approximation ratio of $\mathcal{H}(k)-1 / 2$ for Minimum- $k$-SetCover. Thus, we obtain an improved approximation ratio for constant $k$.
TheOREM 4.6. Let $\mathcal{H}(p)=\sum_{i=1}^{p} \frac{1}{i}$ be the $p$-th harmonic number. For any constant $k \geq 3$, MINIMUM- $k$-ATTRACTOR can be approximated in polynomial time up to a factor of $\mathcal{H}(k(k+1) / 2)-1 / 2$.

\section{OPTIMAL-TIME RANDOM ACCESS}

In this section we show that the simple string attractor property introduced in Definition 3.1 is sufficient to support random access in optimal time on string attractors and, in particular, on most dictionary-compression schemes. We show this fact by extending an existing lower bound of Verbin and Yu [42] (holding on grammars) and by providing a data structure matching this lower bound. First, we reiterate the main step of the proof in [42], with minute technical details tailored to our needs.

THeOREM 5.1 (Verbin AND Yu [42]). Let $g$ be the size of any SLP for a string of length $n$. Any static data structure taking $O(g$ polylog $n)$ space needs $\Omega(\log n / \log \log n)$ time to answer random access queries.

Proof. Consider the following problem: given $m$ points on a grid of size $m \times m^{\epsilon}$, where $\epsilon>0$ is some constant, build a data structure answering 2 -sided parity range-counting queries, i.e., for any position $(x, y)$ find the number (modulo 2 ) of points with coordinates in $[1, x] \times[1, y]$. Any static data structure answering such queries using $O(m$ polylog $m)$ words of space must have a query time of $\Omega(\log m / \log \log m)$ [42, Lem. 5]. Assume that our claim does not hold, i.e., for any SLP of size $g$, there exists a static data structure $D$ of size $O(g$ polylog $n)$ that answers access queries in $o(\log n / \log \log n)$ time. Now take any instance of the above rangecounting problem, i.e., a set of $m$ points on a grid. Take the string of length $n=m^{1+\epsilon}$ encoding answers to all possible queries (call it the answer string) in row-major order. This string, by [42, Lem. 6], has an SLP of size $g \in O(m \log m)$. Thus, $D$ takes $O(g$ polylog $n)=$ $O(m$ polylog $m)$ space and answers access (and hence also rangecounting) queries in $o(\log n / \log \log n)=o(\log m / \log \log m)$ time, contradicting [42, Lem. 5].

The key observation for extending the above lower bound to other compression schemes and to string attractors is that we can use known reductions from SLPs to obtain a different representation (e.g., a collage system or a macro scheme) of size at most $g$. For example, the fact that $z \leq g^{*}$ [39] immediately implies that the above bound also holds within $O(z$ polylog $n)$ space. Hence, for any compression method that is at least as powerful as SLPs we can generalize the lower bound.

Theorem 5.2. Let $T \in \Sigma^{n}$ and let $\alpha$ be any of these measures:

(1) the size $\gamma$ of a string attractor for $T$,

(2) the size $g_{r l}$ of an RLSLP for $T$,

(3) the size $c$ of a collage system for $T$,

(4) the size $z$ of the LZ77 parse of T,

(5) the size $b$ of a macro scheme for $T$.

Then, $\Omega(\log n / \log \log n)$ time is needed to access one random position of $T$ within $O(\alpha$ polylog $n)$ space.

Proof. Let $G$ be the SLP of size $g$ used in Theorem 5.1 to compress the answer string. By our reduction stated in Theorem 3.5, we can build a string attractor of size $\gamma \leq g$, therefore $\gamma$ polylog $n \in$ 
$O(g$ polylog $n)$ and bound (1) holds. Since RLSLPs and collage systems are extensions of SLPs, $G$ is also an RLSLP and a collage system for $T$, hence bounds (2) and (3) hold trivially. From the relation $z \leq g^{*}$ [39] we have that $z$ polylog $n \in O(g$ polylog $n)$, therefore bound (4) holds. Finally, LZ77 is a particular unidirectional parse, and macro schemes are extensions of unidirectional parses, hence bound (5) holds.

We now describe a parametrized data structure based on string attractors matching lower bounds (1-5) of Theorem 5.2. Our result generalizes Block Trees [6] (where blocks are only copied left-toright) and a data structure proposed very recently by Gagie et al. [21] supporting random access on the RLBWT (where only constant out-degree is considered).

Theorem 5.3. Let $T[1 . . n]$ be a string over alphabet $[1 . . \sigma]$, and let $\Gamma$ be a string attractor of size $\gamma$ for $T$. For any integer parameter $\tau \geq 2$, we can store a data structure of $O\left(\gamma \tau \log _{\tau}(n / \gamma)\right) w$-bit words supporting the extraction of any length- $\ell$ substring of $T$ in $O\left(\log _{\tau}(n / \gamma)+\ell \log (\sigma) / w\right)$ time.

Proof. We describe a data structure supporting the extraction of $\alpha=\frac{w \log _{\tau}(n / \gamma)}{\log \sigma}$ packed characters in $O\left(\log _{\tau}(n / \gamma)\right)$ time. To extract a substring of length $\ell$ we divide it into $\lceil\ell / \alpha\rceil$ blocks and extract each block with the proposed data structure. Overall, this will take $O\left((\ell / \alpha+1) \log _{\tau}(n / \gamma)\right)=O\left(\log _{\tau}(n / \gamma)+\ell \log (\sigma) / w\right)$ time.

Our data structure is organized into $O\left(\log _{\tau}(n / \gamma)\right)$ levels. For simplicity, we assume that $\gamma$ divides $n$ and that $n / \gamma$ is a power of $\tau$. The top level (level 0) is special: we divide the string into $\gamma$ blocks $T[1 . . n / \gamma] T[n / \gamma+1 . .2 n / \gamma] \ldots T[n-n / \gamma+1 . . n]$ of size $n / \gamma$. Intuitively, at each level $i>0$ we associate to each $j \in \Gamma$ two context substrings of length $s_{i}=n /\left(\gamma \cdot \tau^{i-1}\right)$ flanking position $j$. These substrings are divided in a certain number of (overlapping) blocks of length $s_{i} / \tau=s_{i+1}$. Each block is then associated to an occurrence at level $i+1$ overlapping some element $j^{\prime} \in \Gamma$ (possible by definition of $\Gamma$ ). At some particular level $i^{*}$ (read the formal description below) we store explicitly all characters in the context substrings. To extract a substring of length $\alpha$, we will map it from level 0 to level $i^{*}$, and then extract naively using the explicitly stored characters.

More formally, for levels $i>0$ and for every element $j \in \Gamma$, we consider the $2 \tau$ non-overlapping blocks of length $s_{i+1}$ forming the two context substrings flanking $j: T\left[j-s_{i+1} \cdot k+1 \ldots j-s_{i+1} \cdot(k-1)\right]$ and $T\left[j+s_{i+1} \cdot(k-1)+1 \ldots j+s_{i+1} \cdot k\right]$, for $k=1, \ldots, \tau$. We moreover consider a sequence of $2 \tau-1$ additional consecutive and non-overlapping blocks of length $s_{i+1}$, starting in the middle of the first block above defined and ending in the middle of the last: $T\left[j-s_{i+1} \cdot k+1+s_{i+1} / 2 \ldots j-s_{i+1} \cdot(k-1)+s_{i+1} / 2\right]$ for $k=1, \ldots, \tau$, and $T\left[j+s_{i+1} \cdot(k-1)+1+s_{i+1} / 2 \ldots j+s_{i+1} \cdot k+s_{i+1} / 2\right]$, for $k=1, \ldots, \tau-1$. Note that, with this choice of blocks, at level $i$ for any substring $S$ of length at most $s_{i+1} / 2$ inside the context substrings around elements of $\Gamma$ we can always find a block fully containing $S$. This property will now be used to map "short" strings from the first to last level of our structure without splitting them, until reaching explicitly stored characters at some level $i^{*}$ (see below).

From the definition of string attractor, blocks at level 0 and each block at level $i>0$ have an occurrence at level $i+1$ crossing some position in $\Gamma$. Such an occurrence can be fully identified by the coordinate $\langle o f f, j\rangle$, for $0 \leq o f f<s_{i+1}$ and $j \in \Gamma$, indicating that the occurrence starts at position $j-$ off. Let $i^{*}$ be the smallest number such that $s_{i^{*}+1}<2 \alpha=\frac{2 w \log _{\tau}(n / \gamma)}{\log \sigma}$. Then $i^{*}$ is the last level of our structure. At this level, we explicitly store a packed string with the characters of the blocks. This uses in total $O\left(\gamma \cdot s_{i^{*}} \log (\sigma) / w\right)=$ $O\left(\gamma \tau \log _{\tau}(n / \gamma)\right)$ words of space. All the blocks at levels $0 \leq i<i^{*}$ store instead the coordinates $\langle o f f, j\rangle$ of their primary occurrence in the next level. At level $i^{*}-1$, these coordinates point inside the strings of explicitly stored characters.

Let $S=T[i . . i+\alpha-1]$ be the substring to be extracted. Note that we can assume $n / \gamma \geq \alpha$; otherwise the whole string can be stored in plain packed form using $n \log (\sigma) / w<\alpha \gamma \log (\sigma) / w \in$ $O\left(\gamma \log _{\tau}(n / \gamma)\right)$ words and we do not need any data structure. It follows that $S$ either spans two blocks at level 0 , or it is contained in a single block. The former case can be solved with two queries of the latter, so we assume, without losing generality, that $S$ is fully contained inside a block at level 0 . To retrieve $S$, we map it down to the next levels (using the stored coordinates) as a contiguous substring as long as this is possible, that is, as long as it fits inside a single block. Note that, thanks to the way blocks overlap, this is always possible as long as level $i$ is such that $\alpha \leq s_{i+1} / 2$. By definition, then, we arrive in this way precisely to level $i^{*}$, where characters are stored explicitly and we can return the packed substring. Note also that, since blocks in the same level have the same length, at each level we spend only constant time to find the pointer to the next level (this requires a simple integer division).

Table 2 reports some interesting space-time trade-offs achievable with our data structure. For $\tau=\log ^{\epsilon} n$, the data structure takes $O(\gamma$ polylog $n)$ space and answers random access queries in $O(\log (n / \gamma) / \log \log n)$ time, which is optimal by Theorem 5.2 (note that $\log (n / \gamma) \in \Theta(\log n)$ for the string used in Theorem 5.2, so the structure does not break the lower bound). Choosing $\tau=(n / \gamma)^{\epsilon}$, space increases to $O\left(\gamma^{1-\epsilon} n^{\epsilon}\right)$ words and query time is optimal in the packed setting. Note that our data structure is universal: given any dictionary-compressed representation, by the reductions of Section 3.1 we can derive a string attractor of the same asymptotic size and build our data structure on top of it. By Theorems 5.1 and 5.2 we obtain:

Corollary 5.4. For $\tau=\log ^{\epsilon} n$ (for any constant $\epsilon>0$ ), the data structure of Theorem 5.3 supports random access in optimal time on string attractors, SLPs, RLSLPs, LZ77, collage systems, and macro schemes.

\section{CONCLUSIONS}

In this paper we have proposed a new theory unifying all known dictionary compression techniques. The new combinatorial object at the core of this theory - the string attractor - is NP-hard to optimize within some constant in polynomial time, but logarithmic approximations can be achieved using compression algorithms and reductions to well-studied combinatorial problems. We have moreover shown a data structure supporting optimal random access queries on string attractors and on most known dictionary compressors. Random access stands at the core of most compressed computation techniques; our results suggest that compressed computation can be performed independently of the underlying compression scheme (and even in optimal time for some queries). 
An interesting view for future research is to treat (the size of the smallest) $k$-attractors as a measure of string compressibility akin to the $k$-th order empirical entropy (which has proven to be an accurate and robust measure for texts that are not highly-repetitive), as it exhibits a similar regularity, e.g., $\gamma_{k}^{*}(X) \leq \gamma_{k+1}^{*}(X)$ for any $k$, while being sensitive to repetition: $\gamma_{k}^{*}\left(X^{t}\right) \leq \gamma_{k}^{*}(X)+1$.

Another use of our techniques could be to provide a linear ordering of compression algorithms based on how well they approximate the smallest attractor. For example, the unary string shows that a "weak" compression like LZ78 in the worst case cannot achieve a better ratio than $|L Z 78| / \gamma^{*} \in \Omega(\sqrt{n})$, while we showed that LZ77 achieves (via our reductions from attractors) $|L Z 77| / \gamma^{*} \in O$ (polylog $n$ ) ratio. Relatedly, it is still an open problem to determine whether the smallest attractor can be approximated up to $o(\log n)$ ratio in polynomial time for all strings. Even within logarithmic ratio, we have left open the problem of efficiently computing such an approximation. A naive implementation of our algorithm based on set-cover runs in cubic time.

It would also be interesting to further explore the landscape of compressed data structures based on string attractors. In this paper we showed that the simple string attractor property is sufficient to support random access. Is this true for more complex queries such as, e.g., indexing?

Finally, an intriguing problem is that of optimal approximation of string $k$-attractors; e.g., what is the complexity of the 2-attractor problem? what is, assuming $\mathrm{P} \neq \mathrm{NP}$, the best approximation ratio for the minimum 3-attractor problem? For the latter question, in this paper we gave a lower bound of 11809/11808 (Corollary 4.4) and an upper bound of 1.95 (Theorem 4.6).

\section{ACKNOWLEDGMENTS}

We received useful suggestions from many people during the writeup of this paper. We would like to thank (in alphabetical order) Philip Bille, Anders Roy Christiansen, Mikko Berggren Ettienne, Travis Gagie, Inge Li Gørtz, Juha Kärkkäinen, Gonzalo Navarro, Alberto Policriti, and Esko Ukkonen for the great feedback.

This work was partially funded by Danish Research Council grant DFF-4005-00267 and by the project MIUR-SIR CMACBioSeq ("Combinatorial methods for analysis and compression of biological sequences”) grant RBSI146R5L.

\section{REFERENCES}

[1] D. Belazzougui. 2014. Linear time construction of compressed text indices in compact space. In STOC. 148-193.

[2] D. Belazzougui, P. Hagge Cording, S. J. Puglisi, and Y. Tabei. 2015. Access, rank, and select in grammar-compressed strings. In ESA. 142-154.

[3] D. Belazzougui and F. Cunial. 2017. Fast label extraction in the CDAWG. In SPIRE. 161-175.

[4] D. Belazzougui and F. Cunial. 2017. Representing the suffix tree with the CDAWG. In CPM. 7:1-7:13.

[5] D. Belazzougui, F. Cunial, T. Gagie, N. Prezza, and M. Raffinot. 2015. Composite repetition-aware data structures. In CPM. 26-39.

[6] D. Belazzougui, T. Gagie, P. Gawrychowski, J. Kärkkäinen, A. O. Pereira, S. J. Puglisi, and Y. Tabei. 2015. Queries on LZ-bounded encodings. In DCC. 83-92.

[7] P. Berman and M. Karpinski. 1999. On some tighter inapproximability results. In ICALP. 200-209.

[8] P. Bille, M. B. Ettienne, I. L. Gørtz, and H. W. Vildhøj. 2017. Time-space trade-offs for Lempel-Ziv compressed indexing. In CPM. 16:1-16:17.

[9] P. Bille, G. M. Landau, R. Raman, K. Sadakane, S. R. Satti, and O. Weimann. 2015 Random access to grammar-compressed strings and trees. SIAM f. Comput. 44, 3 (2015), 513-539.
[10] A. Blumer, J. Blumer, D. Haussler, R. M. McConnell, and A. Ehrenfeucht. 1987. Complete inverted files for efficient text retrieval and analysis. F. ACM 34, 3 (1987), 578-595.

[11] M. Burrows and D. J. Wheeler. 1994. A block-sorting lossless data compression algorithm. Technical Report 124. Digital Equipment Corporation.

[12] M. Charikar, E. Lehman, D. Liu, R. Panigrahy, M. Prabhakaran, A. Sahai, and A. Shelat. 2005. The smallest grammar problem. IEEE Trans. Information Theory 51, 7 (2005), 2554-2576.

[13] T. M. Cover and J. A. Thomas. 2006. Elements of information theory 2nd edition. Wiley.

[14] M. Crochemore and L. Ilie. 2008. Computing Longest Previous Factor in linear time and applications. Inf. Process. Lett. 106, 2 (2008), 75-80.

[15] M. Crochemore and R. Vérin. 1997. Direct construction of compact directed acyclic word graphs. In CPM. 116-129.

[16] M. Crochemore and R. Vérin. 1997. On compact directed acyclic word graphs. In Structures in Logic and Computer Science. Springer, 192-211.

[17] R. Duh and M. Fürer. 1997. Approximation of $k$-set cover by semi-local optimization. In STOC. 256-264.

[18] T. Gagie. 2006. Large alphabets and incompressibility. Inf. Process. Lett. 99, 6 (2006), 246-251.

[19] T. Gagie, G. Navarro, and N. Prezza. 2017. Optimal-time text indexing in BWTruns bounded space. arXiv preprint 1705.10382 (2017)

[20] T. Gagie, G. Navarro, and N. Prezza. 2018. On the approximation ratio of LempelZiv parsing. In LATIN. To appear.

[21] T. Gagie, G. Navarro, and N. Prezza. 2018. Optimal-time text indexing in BWTruns bounded space. In SODA. 1459-1477.

[22] J. K. Gallant. 1982. String compression algorithms. Ph.D. Dissertation. Princeton University.

[23] D. Hucke, M. Lohrey, and C. P. Reh. 2016. The smallest grammar problem revisited. In SPIRE. 35-49.

[24] D. S. Johnson. 1973. Approximation algorithms for combinatorial problems. In STOC. $38-49$.

[25] T. Kida, T. Matsumoto, Y. Shibata, M. Takeda, A. Shinohara, and S. Arikawa. 2003. Collage system: A unifying framework for compressed pattern matching. Theor. Comput. Sci. 298, 1 (2003), 253-272.

[26] J. C. Kieffer and E. Yang. 2000. Grammar-based codes: A new class of universal lossless source codes. IEEE Trans. Information Theory 46, 3 (2000), 737-754.

[27] J. C. Kieffer, E. Yang, G. J. Nelson, and P. C. Cosman. 2000. Universal lossless compression via multilevel pattern matching. IEEE Trans. Information Theory 46, 4 (2000), 1227-1245.

[28] A. N. Kolmogorov. 1965. Three approaches to the quantitative definition of information. Probl. Inf. Transm. 1, 1 (1965), 1-7.

[29] S. R. Kosaraju and G. Manzini. 1999. Compression of low entropy strings with Lempel-Ziv algorithms. SIAM f. Comput. 29, 3 (1999), 893-911.

[30] S. Kreft and G. Navarro. 2013. On compressing and indexing repetitive sequences. Theor. Comput. Sci. 483 (2013), 115-133.

[31] N. J. Larsson and A. Moffat. 2000. Off-line dictionary-based compression. Proc. IEEE 88, 11 (2000), 1722-1732.

[32] A. Lempel and J. Ziv. 1976. On the complexity of finite sequences. IEEE Trans. Information Theory 22, 1 (1976), 75-81.

[33] V. Mäkinen, G. Navarro, J. Sirén, and N. Välimäki. 2010. Storage and retrieval of highly repetitive sequence collections. f. Comput. Biol. 17, 3 (2010), 281-308.

[34] U. Manber and E. W. Myers. 1993. Suffix arrays: A new method for on-line string searches. SIAM J. Comput. 22, 5 (1993), 935-948.

[35] J. I. Munro, G. Navarro, and Y. Nekrich. 2017. Space-efficient construction of compressed indexes in deterministic linear time. In SODA. 408-424.

[36] T. Nishimoto, T. I, S. Inenaga, H. Bannai, and M. Takeda. 2016. Fully dynamic data structure for LCE queries in compressed space. In MFCS. 72:1-72:15.

[37] C. H. Papadimitriou and M. Yannakakis. 1988. Optimization, approximation, and complexity classes (extended abstract). In STOC. 229-234.

[38] M. Rodeh, V. R. Pratt, and S. Even. 1981. Linear algorithm for data compression via string matching. 7. ACM 28, 1 (1981), 16-24.

[39] W. Rytter. 2003. Application of Lempel-Ziv factorization to the approximation of grammar-based compression. Theor. Comput. Sci. 302, 1-3 (2003), 211-222.

[40] J. A. Storer and T. G. Szymanski. 1978. The macro model for data compression. In STOC. 30-39.

[41] J. A. Storer and T. G. Szymanski. 1982. Data compression via textural substitution. f. ACM 29, 4 (1982), 928-951.

[42] E. Verbin and W. Yu. 2013. Data structure lower bounds on random access to grammar-compressed strings. In CPM. 247-258.

[43] P. Weiner. 1973. Linear pattern matching algorithms. In SWAT (FOCS). 1-11.

[44] T. A. Welch. 1984. A technique for high-performance data compression. Computer 6, 17 (1984), 8-19.

[45] J. Ziv and A. Lempel. 1977. A universal algorithm for sequential data compression. IEEE Trans. Information Theory 23, 3 (1977), 337-343.

[46] J. Ziv and A. Lempel. 1978. Compression of individual sequences via variable-rate coding. IEEE Trans. Information Theory 24, 5 (1978), 530-536. 